\title{
OPTIMAL SMALL DATA SCATTERING FOR THE GENERALIZED DERIVATIVE NONLINEAR SCHRÖDINGER EQUATIONS
}

\author{
RUOBING BAI, YIFEI WU*, AND JUN XUE
}

\begin{abstract}
In this work, we consider the following generalized derivative nonlinear Schrödinger equation

$$
i \partial_{t} u+\partial_{x x} u+i|u|^{2 \sigma} \partial_{x} u=0, \quad(t, x) \in \mathbb{R} \times \mathbb{R} .
$$

We prove that when $\sigma \geq 2$, the solution is global and scattering when the initial data is small in $H^{s}(\mathbb{R}), \frac{1}{2} \leq s \leq 1$. Moreover, we show that when $0<\sigma<2$, there exist a class of solitary wave solutions $\left\{\phi_{c}\right\}$ satisfying

$$
\left\|\phi_{c}\right\|_{H^{1}(\mathbb{R})} \rightarrow 0
$$

when $c$ tends to some endpoint, which is against the small data scattering statement. Therefore, in this model, the exponent $\sigma \geq 2$ is optimal for small data scattering. We remark that this exponent is larger than the short range exponent and the Strauss exponent.
\end{abstract}

\section{INTRODUCTION}

In this paper, we consider the small data scattering of the Cauchy problem for the following generalized derivative nonlinear Schrödinger equation (gDNLS)

$$
\left\{\begin{array}{l}
i \partial_{t} u+\partial_{x}^{2} u+i|u|^{2 \sigma} \partial_{x} u=0, \quad(t, x) \in \mathbb{R} \times \mathbb{R} \\
u(x, 0)=\varphi(x) .
\end{array}\right.
$$

Here $\sigma>0, u: \mathbb{R} \rightarrow \mathbb{C}$ is an unknown function.

The generalized derivative nonlinear Schrödinger equation describes the physical phenomenon of Alfvén waves with small but finite amplitude propagating along the magnetic field in cold plasmas (see for example [55]).

The class of solutions to equation (1.1) is invariant under the scaling

$$
u(t, x) \rightarrow u_{\lambda}(t, x)=\lambda^{\frac{1}{2 \sigma}} u\left(\lambda^{2} t, \lambda x\right) \text { for } \lambda>0,
$$

which maps the initial data as

$$
u(0) \rightarrow u_{\lambda}(0):=\lambda^{\frac{1}{2 \sigma}} \varphi(\lambda x) \text { for } \lambda>0 .
$$

2010 Mathematics Subject Classification. Primary 35Q55; Secondary 35B40.

Key words and phrases. generalized derivative nonlinear Schrödinger equation, scattering, small initial data.

RB and YW are partially supported by NSFC 11771325 and 11571118 . YW is also supported by the National Youth Topnotch Talent Support Program in China. JX is supported by the Research Council of Norway (No. 250070).

* Corresponding author.

Journal of Differential Equations, Volume 269, Issue 9, Pages 6422-6447. This paper is the final version. 
Denote

$$
s_{c}=\frac{1}{2}-\frac{1}{2 \sigma}
$$

then the scaling leaves $\dot{H}^{s_{c}}$ norm invariant, that is,

$$
\|u\|_{\dot{H}_{x}^{s_{c}}}=\left\|u_{\lambda}\right\|_{\dot{H}_{x}^{s_{c}}} .
$$

When $\sigma=1$, take a suitable gauge transformation

$$
u(t, x) \rightarrow u(t, x) \exp \left(-\frac{i}{2} \int_{-\infty}^{x}|u(t, y)|^{2} \mathrm{~d} y\right),
$$

then the equation in (1.1) is transformed into the standard derivative nonlinear Schrödinger equation (DNLS)

$$
i \partial_{t} u+\partial_{x}^{2} u+i \partial_{x}\left(|u|^{2} u\right)=0
$$

For $\sigma \neq 1$, (1.1) is regarded as a generalization of (1.3). The well-posedness theory and the long time behavior of the solution for the equation (1.3) have been widely considered by many researchers. For the local well-posedness result, Hayashi and Ozawa [30, 31] proved that equation (1.3) is locally well-posed in the Sobolev space $H^{1}(\mathbb{R})$ (see also the previous works [19,72]). Later, the best result regarding local well-posedness was obtained in $H^{\frac{1}{2}}(\mathbb{R})$ by Takaoka in [69]. Very recently, Mosincat and Yoon [59] proved the unconditional wellposedness in $H^{s}(\mathbb{R}), s>\frac{1}{2}$ (see also Dan, Li and Ning [13] for the previous work in $H^{s}(\mathbb{R}), s>$ $\left.\frac{2}{3}\right)$. Some other results on local well-posedness can be found in [25, 26, 34, 71]. Moreover, Biagioni and Linares [3] proved the equation (1.3) is ill-posed in $H^{s}(\mathbb{R}), s<\frac{1}{2}$. With regard to the theory of global well-posedness, Hayashi and Ozawa 30] proved that it is globally wellposed in $H^{1}(\mathbb{R})$ under the condition that the initial data satisfies $\left\|u_{0}\right\|_{L^{2}}<\sqrt{2 \pi}$. Wu [74, 75] showed that it is globally well-posed in $H^{1}(\mathbb{R})$ under the condition $\left\|u_{0}\right\|_{L^{2}}<2 \sqrt{\pi}$. Guo and $\mathrm{Wu}$ [21] later proved that it is globally well-posed in $H^{\frac{1}{2}}(\mathbb{R})$ under the same condition of initial data (see also [9, 10, 60, 70] for the previous results on the low regularity). The same results also hold in the periodic case (see Mosincat and Oh [58] in $H^{1}(\mathbb{T}$ ), and Mosincat [57] in $\left.H^{\frac{1}{2}}(\mathbb{T})\right)$. More recently, Jenkins, Liu, Perry and Sulem [37] proved that the Cauchy problem (1.3) is globally well-posed in the weighted Sobolev space $H^{2,2}(\mathbb{R})$.

The equation in (1.1) in the case of $\sigma \neq 1$ also attracts a lot of researchers in recent years. Firstly, for the local well-posedness result, when $0<\sigma<\frac{1}{2}$, Linares, Ponce and Santos [46, 47] proved the local well-posedness for a class of data of arbitrary size in an appropriate weighted Sobolev space. When $\frac{1}{2} \leq \sigma<1$, Hayashi and Ozawa 33 proved that (gDNLS) is locally well-posed in $H^{2}(\mathbb{R})$, and Santos [66] showed the local well-posedness in a weighted sobolev space. When $\sigma>1$, Hayashi and Ozawa [33] proved that (gDNLS) is locally well-posed in energy space $H^{1}(\mathbb{R})$. Hao [28] proved that it is locally well-posed in $H^{\frac{1}{2}}(\mathbb{R})$, when $\sigma \geq \frac{5}{2}$. Santos [66] proved that it is locally well-posed in $H^{\frac{1}{2}}(\mathbb{R})$ with small initial data when $\sigma>1$. Secondly, compared with the local well-posedness, there are only few results of global well-posedness. When $0<\sigma<1$, Hayashi and Ozawa [33] showed the global existence without uniqueness of (gDNLS) in $H^{1}(\mathbb{R})$. When $\sigma>1$, Fukaya, Hayashi and Inui [15] gave a sufficient condition of initial data for global well-posedness in $H^{1}(\mathbb{R})$. Some other results related to the stability theory and inverse scattering theory can be found in $[7,8,16,18,20,22,36,38,43,44,49,54,62,63,68$, 61 and the references therein. 
The equation that we investigate in (1.1) in this paper can be also treated as the form of

$$
i \partial_{t} u+\Delta u=P\left(u, \bar{u}, \partial_{x} u, \partial_{x} \bar{u}\right)
$$

The well-posedness theory of equation (1.4) has been studied by many researchers. Here we only introduce some works and readers can seek further literatures from related references, when $P$ is a polynomial of the form $P(z)=\sum_{d \leq|\alpha| \leq l} C_{\alpha} z^{\alpha}$ and $l, d$ are integers with $l \geq d$. For general cases with $d \geq 3$, Kenig, Ponce and Vega [41] showed that the equation (1.4) is locally well-posed with small initial data in $H^{\frac{7}{2}}(\mathbb{R})$. Some further results have been acquired when $P$ is only composed of $\bar{u}$ and $\partial_{x} \bar{u}$ under some suitable assumption. Grünrock [27] proved that the equation (1.4) is locally well-posed for $s>\frac{1}{2}-\frac{1}{d-1}$ when $P=\partial_{x}\left(\bar{u}^{d}\right)$ and $s>\frac{3}{2}-\frac{1}{d-1}$ when $P=\left(\partial_{x} \bar{u}\right)^{d}$ respectively. Hirayama [35] later extended Grünrock's results to the small data global well-posedness for $s \geq \frac{1}{2}-\frac{1}{d-1}$ when $P=\partial_{x}\left(\bar{u}^{d}\right)$. Recently, Pornnopparath 64] proved that when each term in $P$ contains only one derivative, the equation (1.4) is locally well-posed in $H^{\frac{1}{2}}(\mathbb{R})$, and when a term in $P$ has more than one derivative, the equation (1.4) is locally well-posed in $H^{\frac{3}{2}}(\mathbb{R})$. Moreover, Pornnopparath also proved that when $d \geq 5$, (1.4) is almost globally well-posed in $H^{s}(\mathbb{R})$ when $P$ has only one derivative and $s>\frac{1}{2}$, or when $P$ has more than one derivative and $s>\frac{3}{2}$. For higher dimension and more related theories, see [1,2, 11, 41, 73] and the references therein.

All of the results above are related to the theories of local and global well-posedness. To our knowledge, there is no scattering result yet to (gDNLS). The related result on modified scattering can be found in [23, 32] and the references therein.

One of the motivation to prove scattering is that we believe the small data scattering result of the present paper is significative to our further study. In order to consider the longtime behavior of the solution to (gDNLS), the small data scattering theory is initially needed in some situation, for example, long-time perturbation theory when we use the concentrationcompactness argument.

Moreover, it was known that when $\sigma=1$, there exist solitary wave solutions which can be arbitrarily close to zero. This implies that the small data scattering is not true when $\sigma=1$. So one may wonder the optimal value of $\sigma$ such that the scattering statement holds when the initial data is small enough in some Sobolev space. This is another motivation in the present paper.

For semilinear Schrödinger equation, there are two important exponents named short range exponent and the Strauss exponent. When the nonlinear power is larger than the short range exponent 3 ( $1+\frac{2}{d}$ for general dimensions), one has the global well-posedenss and the existence of the wave operator for small data (see for examples [6, 17, 61]); when the nonlinear power is larger than the Strauss exponent $\frac{\sqrt{17}+3}{2} \approx 3.56\left(\frac{\sqrt{d^{2}+12 d+4}+d+2}{2}\right.$ for general dimensions), one has the scattering for small data (see [67]). According to these, especially because of the short range exponent, one may ask whether $\sigma=1$ is the optimal exponent for scattering. However, there is no such general result for non-semilinear Schrödinger equation (related results see [12, 14, 24, 29] and the references therein). In fact, it is of much model dependence when the nonlinearity contains derivatives. In the present paper, as what we will show in the following, the situation for the nonlinear Schrödinger equation with derivatives is of much difference, compared with the semilinear Schrödinger equations, and the models 
mentioned in the references above, the optimal exponent for scattering is 5, which is much larger than the short range exponent and the Strauss exponent.

For all $\sigma>0$, the equation (1.1) has a two-parameter family of solitary waves,

$$
u_{\omega, c}(t)=e^{i \omega t} \phi_{\omega, c}(x-c t),
$$

where the parameters $c^{2}<4 \omega$, and $\phi_{\omega, c}$ is the solution of the form

$$
\phi_{\omega, c}(x)=\varphi_{\omega, c}(x) \exp \left\{\frac{c}{2} i x-\frac{i}{2 \sigma+2} \int_{-\infty}^{x} \varphi_{\omega, c}^{2 \sigma}(y) d y\right\},
$$

with

$$
\varphi_{\omega, c}(x)=\left\{\frac{(\sigma+1)\left(4 \omega-c^{2}\right)}{2 \sqrt{\omega} \cosh \left(\sigma \sqrt{4 \omega-c^{2}} x\right)-c}\right\}^{\frac{1}{2 \sigma}} .
$$

Firstly, we show that there exists a sequence of solitary waves which are arbitrary small in $H^{1}(\mathbb{R})$ when $0<\sigma<2$; while all of the solitary waves are away from the origin when $\sigma \geq 2$. Let $\Omega=\{(\omega, c): c \in(-2 \sqrt{\omega}, 2 \sqrt{\omega})\}$.

Theorem 1.1. Let $\phi_{\omega, c}$ be defined in (1.5), and $(\omega, c) \in \Omega$, then

(1) when $\sigma \in(0,2)$,

$$
\left\|\phi_{\omega, c}\right\|_{H^{1}(\mathbb{R})} \rightarrow 0, \quad \text { when } c \rightarrow-2 \sqrt{\omega}
$$

(2) when $\sigma \geq 2$, there exists a constant $c_{0}=c_{0}(\sigma)>0$, such that for any $(\omega, c) \in \Omega$,

$$
\left\|\phi_{\omega, c}\right\|_{\dot{H}^{s_{c}(\mathbb{R})}} \geq c_{0} .
$$

Hence, the small data scattering is not true for all $0<\sigma<2$, but from (2) in Theorem 1.1, it is reasonable to conjecture that the small data scattering holds when $\sigma \geq 2$. Our second result aims to show this assertion. Note that we can not replace the norm $\dot{H}^{s_{c}}$ by $L^{p_{c}}, p_{c}=2 \sigma$ in Theorem 1.1 (2), although the latter space is also invariant under the scaling (1.2) (see Remark 3.1 below).

Before stating our second main result, we define the working space

$$
\begin{aligned}
\|u\|_{X_{T}}= & \|u\|_{L_{t}^{\infty} H_{x}^{s}([0, T] \times \mathbb{R})}+\left\|\partial_{x} u\right\|_{L_{x}^{\infty} L_{t}^{2}(\mathbb{R} \times[0, T])}+\sup _{q \in\left[4, N_{0}\right]}\|u\|_{L_{x}^{q} L_{t}^{\infty}(\mathbb{R} \times[0, T])} \\
& +\|u\|_{L_{t}^{4} L_{x}^{\infty}([0, T] \times \mathbb{R})}+\left\|D^{s-\frac{1}{2}} u\right\|_{L_{x}^{4} L_{t}^{\infty}(\mathbb{R} \times[0, T])} \\
& +\left\|D^{s-\frac{1}{2}} \partial_{x} u\right\|_{L_{x}^{\infty} L_{t}^{2}(\mathbb{R} \times[0, T])}+\left\|D^{s-\frac{1}{2}} u\right\|_{L_{t}^{4} L_{x}^{\infty}([0, T] \times \mathbb{R})} .
\end{aligned}
$$

Here $N_{0}$ is any fixed arbitrary large parameter. Now our second main result is

Theorem 1.2. Let $\sigma \geq 2, \frac{1}{2} \leq s \leq 1$ and $\varphi \in H^{s}(\mathbb{R})$. Then there exists a constant $\delta_{0}>0$, such that if $\|\varphi\|_{H^{s}(\mathbb{R})} \leq \delta_{0}$, then the corresponding solution $u$ is global, and

$$
\|u\|_{X_{\infty}} \lesssim\|\varphi\|_{H^{s}(\mathbb{R})} .
$$

Moreover, there exists a unique $u_{ \pm}$such that for any $0 \leq s^{\prime}<s$,

$$
\left\|u(t)-e^{i t \Delta} u_{ \pm}\right\|_{H^{s^{\prime}(\mathbb{R})}} \rightarrow 0 \quad \text { as } \quad t \rightarrow \pm \infty .
$$


Remark 1.3. The same result is also true when we consider the nonlinearity $P\left(u, \bar{u}, \partial_{x} u, \partial_{x} \bar{u}\right)$ and $d \geq 5$ in (1.4) with $P$ has only one derivative. As a comparable result, Pornnopparath 64 proved that when $\sigma \geq 2$, and is an integer, the equation in (1.1) is almost globally wellposed in $H^{s}(\mathbb{R}), s>\frac{1}{2}$. Here "almost" is in the sense that given an arbitrary large $T>0$, there exists a constant $C=C(T)>0$, such that for any initial data $u_{0}:\left\|u_{0}\right\|_{H^{s}(\mathbb{R})} \leq C$, the corresponding solution is in $[0, T]$. Theorem 1.2 improves Pornnopparath's result. On one hand, we do not restrict that $\sigma$ is an integer. On the other hand, as a byproduct of scattering, we prove the global well-posedness in $H^{s}(\mathbb{R}), \frac{1}{2} \leq s \leq 1$, which contains the "endpoint" case $s=\frac{1}{2}$ and the global well-posedness in the general sense.

We believe that the index $s=\frac{1}{2}$ is optimal for local well-posedness in the sense of uniform continuity of the solution flow. However, it is not proved in this paper and leaves us an interesting problem to pursue later.

Moreover, it is worth noting that our scattering result is not applicable in $H^{s}$ if the initial data $\varphi \in H^{s}(\mathbb{R})$ since the index $s^{\prime}$ in Theorem 1.2 satisfies $s^{\prime}<s$.

Based on the local well-posedness result of Santos [66, we use the bootstrap argument to prove Theorem 1.2. More precisely, by defining the working space $X_{T}$ as above with any fixed time $T$, our purpose is to show the uniform-in-time estimate:

$$
\|u\|_{X_{T}} \leq C_{1}\|\varphi\|_{H^{s}(\mathbb{R})}+C_{2}\|u\|_{X_{T}}^{2 \sigma+1} .
$$

Here $C_{1}, C_{2}$ are the constants independent of $T$. The tools we use in the present paper are the smoothing effects and the maximal function estimates. Compared with the low power case $\sigma<2$, the maximal function estimates in the case of $\sigma \geq 2$ provide many benefits. This enables us to handle the nonlinearity properly and establish the uniform-in-time estimate. However, since our desired result is stronger than the previous ones, the situation here has more obstacles. The key ingredients in our proofs are presented below.

(1) A suitable working space is constructed. In order to establish the uniform estimation on time $T$, a related complicated working space need to be constructed. We define the working space $\|u\|_{X_{T}}$ in (1.6). We shall prove that the estimation of each norm in $X_{T}$ is closed. The selection of norms plays an important role in our paper.

(2) A key split on the terms involved the fractional derivatives is carried out. The endpoint Kato-Ponce inequality recently proved by Bourgain and Li 4 shall be used to deal with some $L^{\infty}-L^{\infty}$ type Leibniz rule for fractional derivatives. Moreover, a regular process using Hölder's inequality fails to control these terms by $\|u\|_{X_{T}}$, since most of the mixed norms like $\sup _{q \in\left[4, N_{0}\right]}\|u\|_{L_{x}^{q} L_{t}^{\infty}(\mathbb{R} \times[0, T])}$ are the norm of time ahead. So the subtle split is established, thus we are able to change the order of the mixed norm in some applicable way. This idea has significant influence to obtain our whole estimation on the form of $\|u\|_{X_{T}}$.

The rest of the paper is organized as follows. In Section 2, we give some basic notations and some preliminary estimates that will be used throughout in our paper. In Section 3, we prove non-scattering result for (gDNLS) in $H^{1}(\mathbb{R})$ when $\sigma \in(0,2)$. In Section 4 , we prove scattering result for (gDNLS) in $H^{s}(\mathbb{R})$ with small initial datum when $\sigma \geq 2$.

\section{Notation And Preliminary}

2.1. Notation. We write $X \lesssim Y$ or $Y \gtrsim X$ to indicate $X \leq C Y$ for some constant $C>0$. The notation $a+$ denotes $a+\varepsilon$ for any small $\varepsilon$, and also $a-\varepsilon$ for $a-$. Denote $\langle\cdot\rangle=\left(1+|\cdot|^{2}\right)^{\frac{1}{2}}$ 
and $D^{\alpha}=\left(-\partial_{x}^{2}\right)^{\frac{\alpha}{2}}$. The Hilbert space $H^{s}(\mathbb{R})$ is a Banach space of elements such that $\langle\xi\rangle^{s} \hat{u} \in L^{2}(\mathbb{R})$, where $\mathscr{F}$ denotes the Fourier transform $\mathscr{F} u(\xi)=\hat{u}(\xi)=\int_{\mathbb{R}} e^{-2 \pi i x \cdot \xi} u(x) \mathrm{d} x$, and equipped with the norm $\|u\|_{H^{s}}=\left\|\langle\xi\rangle^{s} \hat{u}(\xi)\right\|_{L^{2}}$. We also have an embedding theorem that $\|u\|_{H^{s_{1}}} \lesssim\|u\|_{H^{s_{2}}}$ for any $s_{1} \leq s_{2}, s_{1}, s_{2} \in \mathbb{R}$. Throughout the whole paper, the letter $C$ will denote various positive constants which are of no importance in our analysis. We use the following norms to denote the mixed spaces $L_{t}^{q} L_{x}^{r}([0, T] \times \mathbb{R})$ and $L_{x}^{r} L_{t}^{q}(\mathbb{R} \times[0, T])$, that is,

$$
\|u\|_{L_{t}^{q} L_{x}^{r}([0, T] \times \mathbb{R})}=\left(\int_{0}^{T}\|u\|_{L_{x}^{r}(\mathbb{R})}^{q} \mathrm{~d} t\right)^{\frac{1}{q}}
$$

and

$$
\|u\|_{L_{x}^{r} L_{t}^{q}(\mathbb{R} \times[0, T])}=\left(\int_{\mathbb{R}}\|u\|_{L_{t}^{q}([0, T])}^{r} \mathrm{~d} x\right)^{\frac{1}{r}}
$$

2.2. Preliminary. In this section, we state some preliminary estimates of the linear Schrödinger operator $e^{i t \Delta}$ which will be used in our later sections. Firstly, we recall the well-known Strichartz estimates.

Lemma 2.1. (Strichartz's estimates, see [5]). Let $I \subset \mathbb{R}$ be an interval. For all admissible $\operatorname{pair}\left(q_{j}, r_{j}\right), j=1,2$, satisfying

$$
2 \leq q_{j}, r_{j} \leq \infty \quad \text { and } \quad \frac{2}{q_{j}}=\frac{1}{2}-\frac{1}{r_{j}}
$$

the following estimates hold:

$$
\left\|e^{i t \Delta} f\right\|_{L_{t}^{q_{j}} L_{x}^{r_{j}}(I \times \mathbb{R})} \lesssim\|f\|_{L^{2}(\mathbb{R})}
$$

and

$$
\left\|\int_{0}^{t} e^{i\left(t-t^{\prime}\right) \Delta} F\left(x, t^{\prime}\right) \mathrm{d} t^{\prime}\right\|_{L_{t}^{q_{1}} L_{x}^{r_{1}(I \times \mathbb{R})}} \lesssim\|F\|_{L_{t}^{q_{2}^{\prime}} L_{x}^{r_{2}^{\prime}(I \times \mathbb{R})}},
$$

where $\frac{1}{q_{2}}+\frac{1}{q_{2}^{\prime}}=\frac{1}{r_{2}}+\frac{1}{r_{2}^{\prime}}=1$.

The next lemma is the smoothing effects.

Lemma 2.2. (Smoothing effects, see $[40,48]$ ). Let $I \subset \mathbb{R}$ be an interval, including $I=R$. Then

1)

$$
\left\|D^{\frac{1}{2}} e^{i t \Delta} f\right\|_{L_{x}^{\infty} L_{t}^{2}(\mathbb{R} \times I)} \lesssim\|f\|_{L^{2}(\mathbb{R})}
$$

for all $f \in L^{2}(\mathbb{R})$; and

2)

$$
\left\|D^{\frac{1}{2}} \int_{0}^{t} e^{i\left(t-t^{\prime}\right) \Delta} F\left(x, t^{\prime}\right) \mathrm{d} t^{\prime}\right\|_{L_{t}^{\infty} L_{x}^{2}(I \times \mathbb{R})} \lesssim\|F\|_{L_{x}^{1} L_{t}^{2}(\mathbb{R} \times I)}
$$

3)

$$
\left\|\partial_{x} \int_{0}^{t} e^{i\left(t-t^{\prime}\right) \Delta} F\left(x, t^{\prime}\right) \mathrm{d} t^{\prime}\right\|_{L_{x}^{\infty} L_{t}^{2}(\mathbb{R} \times I)} \lesssim\|F\|_{L_{x}^{1} L_{t}^{2}(\mathbb{R} \times I)} ;
$$

for all $F \in L_{x}^{1} L_{t}^{2}(\mathbb{R} \times I)$. 
Next, we introduce the following maximal function estimates for the linear Schrödinger equation.

Lemma 2.3. (Maximal function estimates, see [39, 42, 56, 65, 66]). Let $I \subset \mathbb{R}$ be an interval. Let $4 \leq p<\infty$ and $s \geq \frac{1}{2}-\frac{1}{p}$. Then we have

$$
\left\|e^{i t \Delta} f\right\|_{L_{x}^{p} L_{t}^{\infty}(\mathbb{R} \times I)} \lesssim\|f\|_{H^{s}(\mathbb{R})}
$$

and

$$
\left\|\int_{0}^{t} e^{i\left(t-t^{\prime}\right) \Delta} F\left(x, t^{\prime}\right) \mathrm{d} t^{\prime}\right\|_{L_{x}^{p} L_{t}^{\infty}(\mathbb{R} \times I)} \lesssim\|F\|_{L_{x}^{1} L_{t}^{2}(\mathbb{R} \times I)}+\|F\|_{L_{t}^{1} L_{x}^{2}(I \times \mathbb{R})} .
$$

Next, we show the Leibniz and chain rule for fractional derivatives, see [4,40,45] and the references therein.

Lemma 2.4. (Leibniz and chain rule for fractional derivatives). Let $I \subset \mathbb{R}$ be an interval. Then

1) Let $s \in(0,1), 1<p \leq \infty$, and $1<p_{1}, p_{2}, p_{3}, p_{4} \leq \infty$ with $\frac{1}{p}=\frac{1}{p_{1}}+\frac{1}{p_{2}}, \frac{1}{p}=\frac{1}{p_{3}}+\frac{1}{p_{4}}$, and let $f, g \in \mathcal{S}(\mathbb{R})$, then

$$
\left\|D^{s}(f g)\right\|_{L^{p}(\mathbb{R})} \lesssim\left\|D^{s} f\right\|_{L^{p_{1}(\mathbb{R})}}\|g\|_{L^{p_{2}(\mathbb{R})}}+\left\|D^{s} g\right\|_{L^{p_{3}(\mathbb{R})}}\|f\|_{L^{p_{4}(\mathbb{R})}} .
$$

2) Let $s \in(0,1)$ and $p, q, p_{1}, p_{2}, q_{2} \in(1, \infty), q_{1} \in(1, \infty]$ such that

$$
\frac{1}{p}=\frac{1}{p_{1}}+\frac{1}{p_{2}} \quad \text { and } \quad \frac{1}{q}=\frac{1}{q_{1}}+\frac{1}{q_{2}} .
$$

Then

$$
\left\|D^{s} F(f)\right\|_{L_{x}^{p} L_{t}^{q}(\mathbb{R} \times I)} \lesssim\left\|F^{\prime}(f)\right\|_{L_{x}^{p_{1}} L_{t}^{q_{1}(\mathbb{R} \times I)}}\left\|D^{s} f\right\|_{L_{x}^{p_{2}} L_{t}^{q_{2}}(\mathbb{R} \times I)}
$$

3) Let $s \in(0,1), s_{1}, s_{2} \in[0, s]$ with $s=s_{1}+s_{2}$. Let $p, p_{1}, p_{2}, q, q_{1}, q_{2} \in(1, \infty)$ be such that

$$
\frac{1}{p}=\frac{1}{p_{1}}+\frac{1}{p_{2}} \text { and } \quad \frac{1}{q}=\frac{1}{q_{1}}+\frac{1}{q_{2}} .
$$

Then

$$
\left\|D^{s}(f g)-f D^{s} g-g D^{s} f\right\|_{L_{x}^{p} L_{t}^{q}(\mathbb{R} \times I)} \lesssim\left\|D^{s_{1}} f\right\|_{L_{x}^{p_{1}} L_{t}^{q_{1}}(\mathbb{R} \times I)}\left\|D^{s_{2}} g\right\|_{L_{x}^{p_{2}} L_{t}^{q_{2}}(\mathbb{R} \times I)} .
$$

Moreover, for $s_{1}=0$ the value $q_{1}=\infty$ is allowed.

4) Let $s \in(0,1), s_{1}, s_{2} \in[0, s]$ with $s=s_{1}+s_{2}$. Let $p_{1}, p_{2}, q_{1}, q_{2} \in(1, \infty)$ with $1=\frac{1}{p_{1}}+\frac{1}{p_{2}}$ and $\frac{1}{2}=\frac{1}{q_{1}}+\frac{1}{q_{2}}$. Then

$$
\left\|D^{s}(f g)-f D^{s} g-g D^{s} f\right\|_{L_{x}^{1} L_{t}^{2}(\mathbb{R} \times I)} \lesssim\left\|D^{s_{1}} f\right\|_{L_{x}^{p_{1}} L_{t}^{q_{1}}(\mathbb{R} \times I)}\left\|D^{s_{2}} g\right\|_{L_{x}^{p_{2}} L_{t}^{q_{2}}(\mathbb{R} \times I)} .
$$




\section{Proof of Theorem 1.1}

In this section, we consider the solitary wave solutions described in Introduction, and give the proof of Theorem 1.1. Let $\Omega=\{(\omega, c): c \in(-2 \sqrt{\omega}, 2 \sqrt{\omega})\}$.

Proof. Note that $\phi_{\omega, c}$ is the solution of the following equation

$$
-\partial_{x}^{2} \phi+\omega \phi+c i \partial_{x} \phi-i|\phi|^{2 \sigma} \partial_{x} \phi=0
$$

Multiplying on both sides with $\overline{x \partial_{x} \phi_{\omega, c}}$, taking the real part and integrating over $\mathbb{R}$, we obtain that for any $(\omega, c) \in \Omega$,

$$
\left\|\partial_{x} \phi_{\omega, c}\right\|_{L^{2}}^{2}=\omega\left\|\phi_{\omega, c}\right\|_{L^{2}}^{2} .
$$

Hence, for the statement (1), we only need to consider $\left\|\phi_{\omega, c}\right\|_{L^{2}(\mathbb{R})}$.

Now we fix $\omega>0$ and denote $\alpha=\sqrt{4 \omega-c^{2}}$. From (1.5), we find that

$$
\begin{aligned}
\int_{\mathbb{R}}\left|\phi_{\omega, c}\right|^{2} \mathrm{~d} x & =\int_{\mathbb{R}}\left|\varphi_{\omega, c}(x)\right|^{2} \mathrm{~d} x \\
& =\int_{\mathbb{R}}\left\{\frac{(\sigma+1)\left(4 \omega-c^{2}\right)}{2 \sqrt{\omega} \cosh \left(\sigma \sqrt{4 \omega-c^{2}} x\right)-c}\right\}^{\frac{1}{\sigma}} \mathrm{d} x \\
& =\left(\frac{\sigma+1}{2 \sqrt{\omega}}\right)^{\frac{1}{\sigma}} \alpha^{\frac{2}{\sigma}} \int_{\mathbb{R}}\left(\frac{1}{\cosh (\sigma \alpha x)-\frac{c}{2 \sqrt{\omega}}}\right)^{\frac{1}{\sigma}} \mathrm{d} x \\
& =\frac{2}{\sigma}\left(\frac{\sigma+1}{2 \sqrt{\omega}}\right)^{\frac{1}{\sigma}} \alpha^{\frac{2}{\sigma}-1} \int_{0}^{\infty}\left(\frac{1}{\cosh x-\frac{c}{2 \sqrt{\omega}}}\right)^{\frac{1}{\sigma}} \mathrm{d} x \\
& =C_{\omega, \sigma} \alpha^{\frac{2}{\sigma}-1} \int_{0}^{\infty}\left(\frac{1}{\cosh x-\frac{c}{2 \sqrt{\omega}}}\right)^{\frac{1}{\sigma}} \mathrm{d} x
\end{aligned}
$$

where $C_{\omega, \sigma}=\frac{2}{\sigma}\left(\frac{\sigma+1}{2 \sqrt{\omega}}\right)^{\frac{1}{\sigma}}$. For convenience, we denote

$$
I(c)=\int_{0}^{\infty}\left(\frac{1}{\cosh x-\frac{c}{2 \sqrt{\omega}}}\right)^{\frac{1}{\sigma}} \mathrm{d} x .
$$

Moreover, we denote $c_{\sigma}$ as

$$
c_{\sigma}=I(-2 \sqrt{\omega})=\int_{0}^{\infty}\left(\frac{1}{\cosh x+1}\right)^{\frac{1}{\sigma}} \mathrm{d} x,
$$

which makes sense since the last integral above is finite. Note that $I(c)$ is an increasing function, thus we have that for any $c:-2 \sqrt{\omega}<c \leq 0$,

$$
c_{\sigma} \leq I(c) \leq I(0) \text {. }
$$

This combining with (3.2) yields that

$$
\int_{\mathbb{R}}\left|\phi_{\omega, c}\right|^{2} \mathrm{~d} x \leq C_{\omega, \sigma} I(0) \alpha^{\frac{2}{\sigma}-1} \rightarrow 0, \quad \text { when } c \rightarrow-2 \sqrt{\omega} \text {. }
$$

This proves the statement (1).

For the statement (2), we split into two cases: $-2 z_{0} \sqrt{\omega} \leq c<2 \sqrt{\omega}$ and $-2 \sqrt{\omega}<c<$ $-2 z_{0} \sqrt{\omega}$. Here $z_{0} \in(0,1)$ is a constant close enough to 1 (one may set $z_{0}=\frac{99}{100}$ ). 
Case 1: $-2 z_{0} \sqrt{\omega} \leq c<2 \sqrt{\omega}$. We denote $p_{c}=2 \sigma$, then by Sobolev's inequality, it reduces to show that there exists a constant $c_{0}>0$, such that for any $(\omega, c) \in \Omega$,

$$
\left\|\phi_{\omega, c}\right\|_{L^{p_{c}(\mathbb{R})} \geq c_{0} .}
$$

From (1.5), we get that

$$
\begin{aligned}
\int_{\mathbb{R}}\left|\phi_{\omega, c}\right|^{p_{c}} \mathrm{~d} x & =\int_{\mathbb{R}}\left|\varphi_{\omega, c}(x)\right|^{2 \sigma} \mathrm{d} x \\
& =\int_{\mathbb{R}} \frac{(\sigma+1)\left(4 \omega-c^{2}\right)}{2 \sqrt{\omega} \cosh \left(\sigma \sqrt{4 \omega-c^{2}} x\right)-c} \mathrm{~d} x \\
& =\frac{\sigma+1}{2 \sqrt{\omega}} \alpha^{2} \int_{\mathbb{R}} \frac{1}{\cosh (\sigma \alpha x)-\frac{c}{2 \sqrt{\omega}}} \mathrm{d} x \\
& =\frac{2(\sigma+1)}{\sigma} \frac{\alpha}{2 \sqrt{\omega}} \int_{0}^{\infty} \frac{1}{\cosh x-\frac{c}{2 \sqrt{\omega}}} \mathrm{d} x .
\end{aligned}
$$

Denote $\beta=\frac{2 \sqrt{\omega}-c}{2 \sqrt{\omega}}$, then $\beta>0$. Hence,

$$
\int_{0}^{\infty} \frac{1}{\cosh x-\frac{c}{2 \sqrt{\omega}}} \mathrm{d} x=\int_{0}^{\infty} \frac{1}{\cosh x-1+\beta} \mathrm{d} x \geq \frac{1}{\beta} .
$$

Hence, this last inequality combining with (3.4) gives that

$$
\int_{\mathbb{R}}\left|\phi_{\omega, c}\right|^{p_{c}} \mathrm{~d} x \geq \frac{2(\sigma+1)}{\sigma} \frac{\alpha}{2 \sqrt{\omega}} \frac{1}{\beta}=\frac{2(\sigma+1)}{\sigma} \sqrt{\frac{2 \sqrt{\omega}+c}{2 \sqrt{\omega}-c}} \geq \frac{2(\sigma+1)}{\sigma} \sqrt{\frac{1-z_{0}}{1+z_{0}}}:=c_{0}^{p_{c}} .
$$

Case 2: $-2 \sqrt{\omega}<c<-2 z_{0} \sqrt{\omega}$. Let $c=-2 z \sqrt{\omega}$, then $z_{0}<z<1$. First, we rewrite $\phi_{\omega, c}, \varphi_{\omega, c}$ in the following forms. Let

$$
h_{z}(x)=\left(\frac{1}{\cosh (2 \sigma x)+z}\right)^{\frac{1}{2 \sigma}} .
$$

Since $z>0$, there exist positive constants $c_{1 \sigma}, C_{j \sigma}, j=1,2,3$ which are independent of $z$, such that

$$
c_{1 \sigma} \leq\left\|h_{z}\right\|_{L^{2}} \leq C_{1 \sigma}, \quad\left\|h_{z}\right\|_{L^{4 \sigma+2}} \leq C_{2 \sigma}, \quad\left\|\partial_{x} h_{z}\right\|_{L^{2}} \leq C_{3 \sigma} .
$$

Moreover, we rewrite

$$
\varphi_{\omega, c}(x)=[2(\sigma+1)]^{\frac{1}{2 \sigma}} \omega^{\frac{1}{4 \sigma}}\left(1-z^{2}\right)^{\frac{1}{2 \sigma}} h_{z}\left(\sqrt{\omega} \sqrt{1-z^{2}} x\right),
$$

and thus

$$
\begin{aligned}
\phi_{\omega, c}(x)=[2(\sigma+1)]^{\frac{1}{2 \sigma}} \omega^{\frac{1}{4 \sigma}}\left(1-z^{2}\right)^{\frac{1}{2 \sigma}} h_{z}\left(\sqrt{\omega} \sqrt{1-z^{2}} x\right) \\
\cdot \exp \left\{-i z \sqrt{\omega} x-i \sqrt{1-z^{2}} \int_{-\infty}^{\sqrt{\omega} \sqrt{1-z^{2}} x} h_{z}^{2 \sigma}(y) d y\right\} .
\end{aligned}
$$

Denote

$$
g_{z}(x)=\left(1-z^{2}\right)^{\frac{1}{2 \sigma}} h_{z}\left(\sqrt{1-z^{2}} x\right) \exp \left\{-i \sqrt{1-z^{2}} \int_{-\infty}^{\sqrt{1-z^{2}} x} h_{z}^{2 \sigma}(y) d y\right\}
$$


then

$$
\phi_{\omega, c}(x)=[2(\sigma+1)]^{\frac{1}{2 \sigma}} \omega^{\frac{1}{4 \sigma}} \exp \{-i z \sqrt{\omega} x\} g_{z}(\sqrt{\omega} x) .
$$

Hence, by scaling, we get that

$$
\left\|\phi_{\omega, c}\right\|_{\dot{H}^{s_{c}}}=[2(\sigma+1)]^{\frac{1}{2 \sigma}}\left\|\exp \{-i z x\} g_{z}\right\|_{\dot{H}^{s_{c}}} .
$$

So it reduces to estimate $\left\|\exp \{-i z x\} g_{z}\right\|_{\dot{H}^{s_{c}}}$. For this, we have

$$
\begin{aligned}
\left\|\exp \{-i z x\} g_{z}\right\|_{\dot{H}^{s_{c}}}^{2} & =\int_{\mathbb{R}}|\xi|^{2 s_{c}}\left|\hat{g}_{z}(\xi+z)\right|^{2} d \xi \\
& =\int_{\mathbb{R}}|\xi-z|^{2 s_{c}}\left|\hat{g}_{z}(\xi)\right|^{2} d \xi \\
& \geq \int_{\left\{|\xi| \leq A_{0} \sqrt{1-z^{2}}\right\}}|\xi-z|^{2 s_{c}}\left|\hat{g}_{z}(\xi)\right|^{2} d \xi
\end{aligned}
$$

where $A_{0}$ is a big constant decided later. Since $0<1-z^{2} \ll 1$, we further get

$$
\left\|\exp \{-i z x\} g_{z}\right\|_{\dot{H}^{s_{c}}}^{2} \geq \frac{1}{2} \int_{\left\{|\xi| \leq A_{0} \sqrt{1-z^{2}}\right\}}\left|\hat{g}_{z}(\xi)\right|^{2} d \xi
$$

Now we claim that by choosing $A_{0}$ large enough,

$$
\int_{\left\{|\xi| \leq A_{0} \sqrt{1-z^{2}}\right\}}\left|\hat{g}_{z}(\xi)\right|^{2} d \xi \geq \frac{1}{2} c_{1 \sigma}^{2}\left(1-z^{2}\right)^{\frac{1}{\sigma}-\frac{1}{2}} .
$$

Indeed, on one hand,

$$
\begin{aligned}
\left\|g_{z}\right\|_{L^{2}} & =\left\|\left(1-z^{2}\right)^{\frac{1}{2 \sigma}} h_{z}\left(\sqrt{1-z^{2}} x\right)\right\|_{L^{2}} \\
& =\left(1-z^{2}\right)^{\frac{1}{2 \sigma}-\frac{1}{4}}\left\|h_{z}\right\|_{L^{2}} \geq c_{1 \sigma}\left(1-z^{2}\right)^{\frac{1}{2 \sigma}-\frac{1}{4}} .
\end{aligned}
$$

On the other hand,

$$
\int_{\left\{|\xi| \geq A_{0} \sqrt{1-z^{2}}\right\}}\left|\hat{g}_{z}(\xi)\right|^{2} d \xi \leq A_{0}^{-2}\left(1-z^{2}\right)^{-1}\left\|\partial_{x} g_{z}\right\|_{L^{2}}^{2}
$$

Moreover, by (3.6), there exists $C_{4 \sigma}>0$ such that

$$
\begin{aligned}
\left\|\partial_{x} g_{z}\right\|_{L^{2}} & \leq\left(1-z^{2}\right)^{\frac{1}{2 \sigma}+1}\left\|h_{z}^{2 \sigma+1}\left(\sqrt{1-z^{2}} x\right)\right\|_{L^{2}}+\left(1-z^{2}\right)^{\frac{1}{2 \sigma}+\frac{1}{2}}\left\|\partial_{x} h_{z}\left(\sqrt{1-z^{2}} x\right)\right\|_{L^{2}} \\
& \leq\left(1-z^{2}\right)^{\frac{1}{2 \sigma}+\frac{3}{4}}\left\|h_{z}\right\|_{L^{4 \sigma+2}}^{2 \sigma+1}+\left(1-z^{2}\right)^{\frac{1}{2 \sigma}+\frac{1}{4}}\left\|\partial_{x} h_{z}\right\|_{L^{2}} \\
& \leq C_{4 \sigma}\left(1-z^{2}\right)^{\frac{1}{2 \sigma}+\frac{1}{4}}
\end{aligned}
$$

Hence,

$$
\int_{\left\{|\xi| \geq A_{0} \sqrt{1-z^{2}}\right\}}\left|\hat{g}_{z}(\xi)\right|^{2} d \xi \leq C_{4 \sigma}^{2} A_{0}^{-2}\left(1-z^{2}\right)^{\frac{1}{\sigma}-\frac{1}{2}} .
$$

Choosing $A_{0}=\sqrt{2} c_{1 \sigma}^{-1} C_{4 \sigma}$, then the last estimate above combining with (3.10) gives the claim (3.9). Thus combining with (3.9) and (3.8), we get

$$
\left\|\exp \{-i z x\} g_{z}\right\|_{\dot{H}^{s_{c}}} \geq \frac{1}{2} c_{1 \sigma}\left(1-z^{2}\right)^{\frac{1}{2 \sigma}-\frac{1}{4}} \text {. }
$$


Now together with the last estimates above and (3.7), and noting that $\sigma \geq 2$ and $1-z^{2} \leq$ $1-z_{0}^{2}$, we obtain that

$$
\left\|\phi_{\omega, c}\right\|_{\dot{H}^{s_{c}}} \geq \frac{1}{2}[2(\sigma+1)]^{\frac{1}{2 \sigma}} c_{1 \sigma}\left(1-z_{0}^{2}\right)^{\frac{1}{2 \sigma}-\frac{1}{4}} .
$$

Therefore, we establish the desired result in the second case. This proves the Theorem 1.1.

Remark 3.1. One may find from the computation above that for any $(\omega, c) \in \Omega$ and $c<0$, there exists some $c_{\sigma}>0$ such that

$$
\int_{\mathbb{R}}\left|\phi_{\omega, c}\right|^{p_{c}} \mathrm{~d} x \leq c_{\sigma} \frac{\alpha}{2 \sqrt{\omega}}
$$

Hence, for any $\sigma>0$,

$$
\int_{\mathbb{R}}\left|\phi_{\omega, c}\right|^{p_{c}} \mathrm{~d} x \rightarrow 0, \quad \text { as } c \rightarrow-2 \sqrt{\omega} .
$$

Therefore, we can not replace $\dot{H}^{s_{c}}$ norm by $L^{p_{c}}$ norm in Theorem 1.1 (2). This may be helpful to understand the structure of the equation.

\section{Proof of Theorem 1.2}

In this section, we give the proof of Theorem 1.2. Given $s \geq \frac{1}{2}$ and $\varphi \in H^{s}(\mathbb{R})$. Recall the locally well-posed result of Santos [66], that is, $u \in C\left([0, T] ; H^{\frac{1}{2}}(\mathbb{R})\right)$ for sufficient small initial data in the case of $\sigma>1$ for (1.1). Based on this, fixing $T>0$, we only need to show the uniform-on-time estimate (1.7). Then the bootstrap argument yields that there exists $\delta_{0}>0$, such that when $\|\varphi\|_{H^{s}(\mathbb{R})} \leq \delta_{0}$,

$$
\|u\|_{X_{T}} \lesssim\|\varphi\|_{H^{s}(\mathbb{R})}
$$

for any $T \in \mathbb{R}$. In the following, we only consider the positive time. Since the negative time direction can be obtained in the same way.

To show (1.7), according to the definition of $\|u\|_{X_{T}}$, we control the norms in the righthand side of (1.6) one by one.

4.1. Estimates on $\|u\|_{L_{t}^{\infty} H_{x}^{s}([0, T] \times \mathbb{R})}$. In this subsection, we give a priori estimate of the solution in $H^{s}$, which is important for global well-posedness. Moreover, one may find that its proof also plays a crucial role in the proof of scattering in the end of the section. Before stepping into the complicated details, we give some remark here. It is worth noting that the maximal function estimate in Lemma 2.3 has both local $(p<4)$ and global results $(p \geq 4)$, see [65. The local version was heavily relied on in previous papers to establish the local well-posedness, see for examples [64,66]. Unfortunately, we emphasize that to obtain the global well-posedness, the local version can not be used in our estimate.

The main result in this subsection is

$$
\|u\|_{L_{t}^{\infty} H_{x}^{s}([0, T] \times \mathbb{R})} \lesssim\|\varphi\|_{H_{x}^{s}(\mathbb{R})}+\|u\|_{X_{T}}^{2 \sigma+1} .
$$

We prove (4.1) by the following two steps.

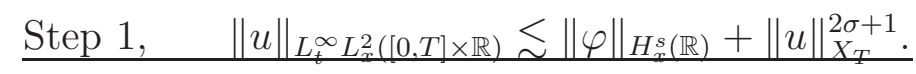


Using the Duhamel formula

$$
u(t)=e^{i t \Delta} \varphi-\int_{0}^{t} e^{i\left(t-t^{\prime}\right) \Delta}\left(|u|^{2 \sigma} \partial_{x} u\right)\left(t^{\prime}\right) \mathrm{d} t^{\prime},
$$

and the Strichartz estimates (2.1) and (2.2), we get

$$
\begin{aligned}
\|u\|_{L_{t}^{\infty} L_{x}^{2}([0, T] \times \mathbb{R})} & \lesssim\left\|e^{i t \Delta} \varphi\right\|_{L_{t}^{\infty} L_{x}^{2}([0, T] \times \mathbb{R})}+\left\|\int_{0}^{t} e^{i\left(t-t^{\prime}\right) \Delta}\left(|u|^{2 \sigma} \partial_{x} u\right)\left(t^{\prime}\right) \mathrm{d} t^{\prime}\right\|_{L_{t}^{\infty} L_{x}^{2}([0, T] \times \mathbb{R})} \\
& \lesssim\|\varphi\|_{L_{x}^{2}(\mathbb{R})}+\left\||u|^{2 \sigma} \partial_{x} u\right\|_{L_{t}^{1} L_{x}^{2}([0, T] \times \mathbb{R})}
\end{aligned}
$$

Next we consider the term $\left\||u|^{2 \sigma} \partial_{x} u\right\|_{L_{t}^{1} L_{x}^{2}([0, T] \times \mathbb{R})}$. We claim that

$$
\left\||u|^{2 \sigma} \partial_{x} u\right\|_{L_{t}^{1} L_{x}^{2}([0, T] \times \mathbb{R})} \lesssim\|u\|_{X_{T}}^{2 \sigma+1}
$$

Now we write

$$
\left\||u|^{2 \sigma} \partial_{x} u\right\|_{L_{t}^{1} L_{x}^{2}([0, T] \times \mathbb{R})}=\left\||u|^{2} \cdot|u|^{2 \sigma-2} \partial_{x} u\right\|_{L_{t}^{1} L_{x}^{2}([0, T] \times \mathbb{R})} .
$$

We consider the inner integration $L_{x}^{2}$ first. By Hölder's inequality, we have

$$
\left\||u|^{2} \cdot|u|^{2 \sigma-2} \partial_{x} u\right\|_{L_{x}^{2}(\mathbb{R})} \lesssim\|u\|_{L_{x}^{\infty}(\mathbb{R})}^{2} \cdot\left\||u|^{2 \sigma-2} \partial_{x} u\right\|_{L_{x}^{2}(\mathbb{R})} .
$$

Hence,

$$
\begin{aligned}
\left\||u|^{2 \sigma} \partial_{x} u\right\|_{L_{t}^{1} L_{x}^{2}([0, T] \times \mathbb{R})} & \lesssim\|\| u\left\|_{L_{x}^{\infty}(\mathbb{R})}^{2} \cdot\right\||u|^{2 \sigma-2} \partial_{x} u\left\|_{L_{x}^{2}(\mathbb{R})}\right\|_{L_{t}^{1}([0, T])} \\
& \lesssim\|u\|_{L_{t}^{4} L_{x}^{\infty}([0, T] \times \mathbb{R})}^{2} \cdot\left\||u|^{2 \sigma-2} \partial_{x} u\right\|_{L_{x}^{2} L_{t}^{2}(\mathbb{R} \times[0, T])}
\end{aligned}
$$

For the term $\left\||u|^{2 \sigma-2} \partial_{x} u\right\|_{L_{x}^{2} L_{t}^{2}(\mathbb{R} \times[0, T])}$, note that $2(2 \sigma-2) \geq 4$, by Hölder's inequality again we obtain

$$
\begin{aligned}
\left\||u|^{2 \sigma-2} \partial_{x} u\right\|_{L_{x}^{2} L_{t}^{2}(\mathbb{R} \times[0, T])} & \lesssim\|u\|_{L_{x}^{2(2 \sigma-2)} L_{t}^{\infty}(\mathbb{R} \times[0, T])}^{2 \sigma-2} \cdot\left\|\partial_{x} u\right\|_{L_{x}^{\infty} L_{t}^{2}(\mathbb{R} \times[0, T])} \\
& \lesssim\|u\|_{X_{T}^{2 \sigma-1}} .
\end{aligned}
$$

Putting this result into (4.6), we get

$$
\begin{aligned}
\left\||u|^{2 \sigma} \partial_{x} u\right\|_{L_{t}^{1} L_{x}^{2}([0, T] \times \mathbb{R})} & \lesssim\|u\|_{L_{t}^{4} L_{x}^{\infty}([0, T] \times \mathbb{R})}^{2} \cdot\|u\|_{X_{T}}^{2 \sigma-1} \\
& \lesssim\|u\|_{X_{T}}^{2 \sigma+1} .
\end{aligned}
$$

Thus we have proved claim (4.4). Then by (4.3), we have

$$
\|u\|_{L_{t}^{\infty} L_{x}^{2}([0, T] \times \mathbb{R})} \lesssim\|\varphi\|_{H_{x}^{s}(\mathbb{R})}+\|u\|_{X_{T}}^{2 \sigma+1}
$$

Thus we have finished the proof on Step 1.

Step 2, $\quad\left\|D^{s} u\right\|_{L_{t}^{\infty} L_{x}^{2}([0, T] \times \mathbb{R})} \lesssim\|\varphi\|_{H_{x}^{s}(\mathbb{R})}+\|u\|_{X_{T}}^{2 \sigma+1}$. 
Using the Duhamel formula (4.2) and the Strichartz estimate (2.1), the smoothing effect (2.4), we have

$$
\begin{aligned}
\left\|D^{s} u\right\|_{L_{t}^{\infty} L_{x}^{2}([0, T] \times \mathbb{R})} & \lesssim\left\|e^{i t \Delta} D^{s} \varphi\right\|_{L_{t}^{\infty} L_{x}^{2}([0, T] \times \mathbb{R})}+\left\|D^{\frac{1}{2}} \int_{0}^{t} e^{i\left(t-t^{\prime}\right) \Delta} D^{s-\frac{1}{2}}\left(|u|^{2 \sigma} \partial_{x} u\right) \mathrm{d} t^{\prime}\right\|_{L_{t}^{\infty} L_{x}^{2}([0, T] \times \mathbb{R})} \\
& \lesssim\left\|D^{s} \varphi\right\|_{L_{x}^{2}(\mathbb{R})}+\left\|D^{s-\frac{1}{2}}\left(|u|^{2 \sigma} \partial_{x} u\right)\right\|_{L_{x}^{1} L_{t}^{2}(\mathbb{R} \times[0, T])} \\
& \lesssim\|\varphi\|_{H_{x}^{s}(\mathbb{R})}+\left\|D^{s-\frac{1}{2}}\left(|u|^{2 \sigma} \partial_{x} u\right)\right\|_{L_{x}^{1} L_{t}^{2}(\mathbb{R} \times[0, T])}
\end{aligned}
$$

Next we claim that

$$
\left\|D^{s-\frac{1}{2}}\left(|u|^{2 \sigma} \partial_{x} u\right)\right\|_{L_{x}^{1} L_{t}^{2}(\mathbb{R} \times[0, T])} \lesssim\|u\|_{X_{T}}^{2 \sigma+1}
$$

To prove this claim, we split it into two cases: $s=\frac{1}{2}$ and $\frac{1}{2}<s \leq 1$.

Case 1: $s=\frac{1}{2}$.

By Hölder's inequality, note that $2 \sigma \geq 4$, we have

$$
\begin{aligned}
\left\||u|^{2 \sigma} \partial_{x} u\right\|_{L_{x}^{1} L_{t}^{2}(\mathbb{R} \times[0, T])} & \lesssim\|\| u\left\|_{L_{t}^{\infty}([0, T])}^{2 \sigma} \cdot\right\| \partial_{x} u\left\|_{L_{t}^{2}([0, T])}\right\|_{L_{x}^{1}(\mathbb{R})} \\
& \lesssim\|u\|_{L_{x}^{2 \sigma} L_{t}^{\infty}(\mathbb{R} \times[0, T])}^{2 \sigma} \cdot\left\|\partial_{x} u\right\|_{L_{x}^{\infty} L_{t}^{2}(\mathbb{R} \times[0, T])} \\
& \lesssim\|u\|_{X_{T}}^{2 \sigma+1}
\end{aligned}
$$

Case 2: $\frac{1}{2}<s \leq 1$.

By the Leibniz rule for fractional derivative (2.11), we get

$$
\begin{gathered}
\left\|D^{s-\frac{1}{2}}\left(|u|^{2 \sigma} \partial_{x} u\right)\right\|_{L_{x}^{1} L_{t}^{2}(\mathbb{R} \times[0, T])} \\
\lesssim\left\|D^{s-\frac{1}{2}}\left(|u|^{2 \sigma}\right) \cdot \partial_{x} u\right\|_{L_{x}^{1} L_{t}^{2}(\mathbb{R} \times[0, T])}+\left\||u|^{2 \sigma} \cdot D^{s-\frac{1}{2}} \partial_{x} u\right\|_{L_{x}^{1} L_{t}^{2}(\mathbb{R} \times[0, T])} \\
\quad+\left\|D^{s-\frac{1}{2}}\left(|u|^{2 \sigma}\right)\right\|_{L_{x}^{1+} L_{t}^{\infty-}(\mathbb{R} \times[0, T])} \cdot\left\|\partial_{x} u\right\|_{L_{x}^{\infty-} L_{t}^{2+}(\mathbb{R} \times[0, T])}
\end{gathered}
$$

We estimate on terms above one by one. have

For the first term $\left\|D^{s-\frac{1}{2}}\left(|u|^{2 \sigma}\right) \cdot \partial_{x} u\right\|_{L_{x}^{1} L_{t}^{2}(\mathbb{R} \times[0, T])}$ in (4.13), by Hölder's inequality, we

$$
\begin{aligned}
& \left\|D^{s-\frac{1}{2}}\left(|u|^{2 \sigma}\right) \cdot \partial_{x} u\right\|_{L_{x}^{1} L_{t}^{2}(\mathbb{R} \times[0, T])} \\
\lesssim & \|\| D^{s-\frac{1}{2}}\left(|u|^{2 \sigma}\right)\left\|_{L_{t}^{\infty-}([0, T])} \cdot\right\| \partial_{x} u\left\|_{L_{t}^{2+}([0, T])}\right\|_{L_{x}^{1}(\mathbb{R})} \\
\lesssim & \left\|D^{s-\frac{1}{2}}\left(|u|^{2 \sigma}\right)\right\|_{L_{x}^{1+} L_{t}^{\infty-}(\mathbb{R} \times[0, T])} \cdot\left\|\partial_{x} u\right\|_{L_{x}^{\infty-} L_{t}^{2+}(\mathbb{R} \times[0, T])} \cdot
\end{aligned}
$$


To the term $\left\|D^{s-\frac{1}{2}}\left(|u|^{2 \sigma}\right)\right\|_{L_{x}^{1+} L_{t}^{\infty-}(\mathbb{R} \times[0, T])}$ in (4.14), using (2.9)), note that $\frac{4}{3}(2 \sigma-1) \geq 4$, we get

$$
\begin{aligned}
\left\|D^{s-\frac{1}{2}}\left(|u|^{2 \sigma}\right)\right\|_{L_{x}^{1+} L_{t}^{\infty-}(\mathbb{R} \times[0, T])} & \lesssim\left\||u|^{2 \sigma-1}\right\|_{L_{x}^{\frac{4}{3} L_{t}^{\infty}(\mathbb{R} \times[0, T])}} \cdot\left\|D^{s-\frac{1}{2}} u\right\|_{L_{x}^{4+} L_{t}^{\infty-}(\mathbb{R} \times[0, T])} \\
& \lesssim\|u\|_{X_{T}^{2 \sigma-1}} \cdot\left\|D^{s-\frac{1}{2}} u\right\|_{L_{x}^{4+} L_{t}^{\infty-}(\mathbb{R} \times[0, T])}
\end{aligned}
$$

By interpolating between $\left\|D^{s-\frac{1}{2}} \partial_{x} u\right\|_{L_{x}^{\infty} L_{t}^{2}(\mathbb{R} \times[0, T])}$ and $\|u\|_{L_{x}^{4} L_{t}^{\infty}(\mathbb{R} \times[0, T])}$, we have that for some $\theta_{1} \in(0,1)$,

$$
\begin{aligned}
\left\|D^{s-\frac{1}{2}} u\right\|_{L_{x}^{4+} L_{t}^{\infty-}(\mathbb{R} \times[0, T])} & \lesssim\left\|D^{s-\frac{1}{2}} \partial_{x} u\right\|_{L_{x}^{\infty} L_{t}^{2}(\mathbb{R} \times[0, T])}^{\theta_{1}} \cdot\|u\|_{L_{x}^{4} L_{t}^{\infty}(\mathbb{R} \times[0, T])}^{1-\theta_{1}} \\
& \lesssim\|u\|_{X_{T} .}
\end{aligned}
$$

Then

$$
\left\|D^{s-\frac{1}{2}}\left(|u|^{2 \sigma}\right)\right\|_{L_{x}^{1+} L_{t}^{\infty-}(\mathbb{R} \times[0, T])} \lesssim\|u\|_{X_{T}}^{2 \sigma} .
$$

To the term $\left\|\partial_{x} u\right\|_{L_{x}^{\infty-} L_{t}^{2+}(\mathbb{R} \times[0, T])}$ in (4.14), it follows from the interpolation between $\left\|D^{s-\frac{1}{2}} \partial_{x} u\right\|_{L_{x}^{\infty} L_{t}^{2}(\mathbb{R} \times[0, T])}$ and $\left\|D^{s-\frac{1}{2}} u\right\|_{L_{x}^{4} L_{t}^{\infty}(\mathbb{R} \times[0, T])}$, that is, there exists $\theta_{2} \in(0,1)$,

$$
\begin{aligned}
\left\|\partial_{x} u\right\|_{L_{x}^{\infty-} L_{t}^{2+}(\mathbb{R} \times[0, T])} & \lesssim\left\|D^{s-\frac{1}{2}} \partial_{x} u\right\|_{L_{x}^{\infty} L_{t}^{2}(\mathbb{R} \times[0, T])}^{\theta_{2}} \cdot\left\|D^{s-\frac{1}{2}} u\right\|_{L_{x}^{4} L_{t}^{\infty}(\mathbb{R} \times[0, T])}^{1-\theta_{2}} \\
& \lesssim\|u\|_{X_{T}} .
\end{aligned}
$$

Inserting (4.16) and (4.17) into (4.14), we have

$$
\left\|D^{s-\frac{1}{2}}\left(|u|^{2 \sigma}\right) \cdot \partial_{x} u\right\|_{L_{x}^{1} L_{t}^{2}(\mathbb{R} \times[0, T])} \lesssim\|u\|_{X_{T}}^{2 \sigma+1} .
$$

Thus we complete the estimate on the first term of (4.13).

For the second term $\left\||u|^{2 \sigma} \cdot D^{s-\frac{1}{2}} \partial_{x} u\right\|_{L_{x}^{1} L_{t}^{2}(\mathbb{R} \times[0, T])}$ in (4.13), by Hölder's inequality, note that $2 \sigma \geq 4$, we have

$$
\begin{aligned}
\left\||u|^{2 \sigma} \cdot D^{s-\frac{1}{2}} \partial_{x} u\right\|_{L_{x}^{1} L_{t}^{2}(\mathbb{R} \times[0, T])} & \lesssim\|u\|_{L_{t}^{\infty}([0, T])}^{2 \sigma} \cdot\left\|D^{s-\frac{1}{2}} \partial_{x} u\right\|_{L_{t}^{2}([0, T])} \|_{L_{x}^{1}(\mathbb{R})} \\
& \lesssim\|u\|_{L_{x}^{2 \sigma} L_{t}^{\infty}(\mathbb{R} \times[0, T])}^{2 \sigma} \cdot\left\|D^{s-\frac{1}{2}} \partial_{x} u\right\|_{L_{x}^{\infty} L_{t}^{2}(\mathbb{R} \times[0, T])} \\
& \lesssim\|u\|_{X_{T}^{2 \sigma+1}}^{2 \sigma} .
\end{aligned}
$$

Thus the estimate on the second term of (4.13) is also completed.

For the third term $\left\|D^{s-\frac{1}{2}}\left(|u|^{2 \sigma}\right)\right\|_{L_{x}^{1+} L_{t}^{\infty-}(\mathbb{R} \times[0, T])} \cdot\left\|\partial_{x} u\right\|_{L_{x}^{\infty-} L_{t}^{2+}(\mathbb{R} \times[0, T])}$ in (4.13), using (4.16) and (4.17), we have

$$
\left\|D^{s-\frac{1}{2}}\left(|u|^{2 \sigma}\right)\right\|_{L_{x}^{1+} L_{t}^{\infty-}(\mathbb{R} \times[0, T])} \cdot\left\|\partial_{x} u\right\|_{L_{x}^{\infty-} L_{t}^{2+}(\mathbb{R} \times[0, T])} \lesssim\|u\|_{X_{T}}^{2 \sigma+1}
$$

Inserting (4.18), (4.19) and (4.20) into (4.13), we have

$$
\left\|D^{s-\frac{1}{2}}\left(|u|^{2 \sigma} \partial_{x} u\right)\right\|_{L_{x}^{1} L_{t}^{2}(\mathbb{R} \times[0, T])} \lesssim\|u\|_{X_{T}}^{2 \sigma+1}
$$

Owing to the above two cases, we finish the proof of claim (4.11). Putting (4.21) into (4.10), we finish the proof on Step 2. 
4.2. Estimates on $\left\|\partial_{x} u\right\|_{L_{x}^{\infty} L_{t}^{2}(\mathbb{R} \times[0, T])}$. Using the Duhamel formula (4.2) and the smoothing effects (2.3) and (2.5), we get

$$
\begin{aligned}
\left\|\partial_{x} u\right\|_{L_{x}^{\infty} L_{t}^{2}(\mathbb{R} \times[0, T])} & \lesssim\left\|e^{i t \Delta} \partial_{x} \varphi\right\|_{L_{x}^{\infty} L_{t}^{2}(\mathbb{R} \times[0, T])}+\left\|\partial_{x} \int_{0}^{t} e^{i\left(t-t^{\prime}\right) \Delta}\left(|u|^{2 \sigma} \partial_{x} u\right)\left(t^{\prime}\right) \mathrm{d} t^{\prime}\right\|_{L_{x}^{\infty} L_{t}^{2}(\mathbb{R} \times[0, T])} \\
& \lesssim\|\varphi\|_{H_{x}^{\frac{1}{2}(\mathbb{R})}}+\left\||u|^{2 \sigma} \partial_{x} u\right\|_{L_{x}^{1} L_{t}^{2}(\mathbb{R} \times[0, T])}
\end{aligned}
$$

By (4.12), we have

$$
\left\|\partial_{x} u\right\|_{L_{x}^{\infty} L_{t}^{2}(\mathbb{R} \times[0, T])} \lesssim\|\varphi\|_{H_{x}^{s}(\mathbb{R})}+\|u\|_{X_{T}}^{2 \sigma+1}
$$

4.3. Estimates on $\sup _{q \in\left[4, N_{0}\right]}\|u\|_{L_{x}^{q} L_{t}^{\infty}(\mathbb{R} \times[0, T])}$. By Duhamel's formula (4.2) and the maximal function estimates (2.6) and (2.7), we have

$$
\begin{aligned}
\|u\|_{L_{x}^{q} L_{t}^{\infty}(\mathbb{R} \times[0, T])} & \lesssim\left\|e^{i t \Delta} \varphi\right\|_{L_{x}^{q} L_{t}^{\infty}(\mathbb{R} \times[0, T])}+\left\|\int_{0}^{t} e^{i\left(t-t^{\prime}\right) \Delta}\left(|u|^{2 \sigma} \partial_{x} u\right)\left(t^{\prime}\right) \mathrm{d} t^{\prime}\right\|_{L_{x}^{q} L_{t}^{\infty}(\mathbb{R} \times[0, T])} \\
& \lesssim\|\varphi\|_{H_{x}^{s}(\mathbb{R})}+\left\||u|^{2 \sigma} \partial_{x} u\right\|_{L_{x}^{1} L_{t}^{2}(\mathbb{R} \times[0, T])}+\left\||u|^{2 \sigma} \partial_{x} u\right\|_{L_{t}^{1} L_{x}^{2}([0, T] \times \mathbb{R})},
\end{aligned}
$$

where we have used the condition $s \geq \frac{1}{2} \geq \frac{1}{2}-\frac{1}{q}$ in Lemma 2.3. By (4.4) and (4.12), we obtain

$$
\sup _{q \in\left[4, N_{0}\right]}\|u\|_{L_{x}^{q} L_{t}^{\infty}(\mathbb{R} \times[0, T])} \lesssim\|\varphi\|_{H_{x}^{s}(\mathbb{R})}+\|u\|_{X_{T}}^{2 \sigma+1}
$$

4.4. Estimates on $\|u\|_{L_{t}^{4} L_{x}^{\infty}([0, T] \times \mathbb{R})}$. By Duhamel's formula (4.2) and the Strichartz estimates (2.1) and (2.2), we get

$$
\begin{aligned}
\|u\|_{L_{t}^{4} L_{x}^{\infty}([0, T] \times \mathbb{R})} & \lesssim\left\|e^{i t \Delta} \varphi\right\|_{L_{t}^{4} L_{x}^{\infty}([0, T] \times \mathbb{R})}+\left\|\int_{0}^{t} e^{i\left(t-t^{\prime}\right) \Delta}\left(|u|^{2 \sigma} \partial_{x} u\right)\left(t^{\prime}\right) \mathrm{d} t^{\prime}\right\|_{L_{t}^{4} L_{x}^{\infty}([0, T] \times \mathbb{R})} \\
& \lesssim\|\varphi\|_{L_{x}^{2}(\mathbb{R})}+\left\||u|^{2 \sigma} \partial_{x} u\right\|_{L_{t}^{1} L_{x}^{2}([0, T] \times \mathbb{R})}
\end{aligned}
$$

By (4.4), we have

$$
\|u\|_{L_{t}^{4} L_{x}^{\infty}([0, T] \times \mathbb{R})} \lesssim\|\varphi\|_{L_{x}^{2}(\mathbb{R})}+\|u\|_{X_{T}}^{2 \sigma+1}
$$

4.5. Estimates on $\left\|D^{s-\frac{1}{2}} u\right\|_{L_{x}^{4} L_{t}^{\infty}(\mathbb{R} \times[0, T])}$. It is worth noting that the endpoint Kato-Ponce inequality in 4 plays a significant role in our estimates in which we meet the Leibniz rule for fractional derivatives in $L^{\infty}$. Thanks to this inequality, we are able to deal with the term $\left\|D^{s-\frac{1}{2}}\left(|u|^{2}\right)\right\|_{L_{x}^{\infty}(\mathbb{R})}$. 
Using Duhamel's formula (4.2) and the maximal function estimates (2.6) and (2.7), we get

$$
\begin{aligned}
& \left\|D^{s-\frac{1}{2}} u\right\|_{L_{x}^{4} L_{t}^{\infty}(\mathbb{R} \times[0, T])} \lesssim\left\|e^{i t \Delta} D^{s-\frac{1}{2}} \varphi\right\|_{L_{x}^{4} L_{t}^{\infty}(\mathbb{R} \times[0, T])} \\
& +\left\|\int_{0}^{t} e^{i\left(t-t^{\prime}\right) \Delta} D^{s-\frac{1}{2}}\left(|u|^{2 \sigma} \partial_{x} u\right)\left(t^{\prime}\right) \mathrm{d} t^{\prime}\right\|_{L_{x}^{4} L_{t}^{\infty}(\mathbb{R} \times[0, T])} \\
& \lesssim\left\|D^{s-\frac{1}{2}} \varphi\right\|_{H_{x}^{\frac{1}{2}}(\mathbb{R})}+\left\|D^{s-\frac{1}{2}}\left(|u|^{2 \sigma} \partial_{x} u\right)\right\|_{L_{x}^{1} L_{t}^{2}(\mathbb{R} \times[0, T])} \\
& +\left\|D^{s-\frac{1}{2}}\left(|u|^{2 \sigma} \partial_{x} u\right)\right\|_{L_{t}^{1} L_{x}^{2}([0, T] \times \mathbb{R})}, \\
& \lesssim\|\varphi\|_{H_{x}^{s}(\mathbb{R})}+\left\|D^{s-\frac{1}{2}}\left(|u|^{2 \sigma} \partial_{x} u\right)\right\|_{L_{x}^{1} L_{t}^{2}(\mathbb{R} \times[0, T])} \\
& +\left\|D^{s-\frac{1}{2}}\left(|u|^{2 \sigma} \partial_{x} u\right)\right\|_{L_{t}^{1} L_{x}^{2}([0, T] \times \mathbb{R})} .
\end{aligned}
$$

Recall that the term $\left\|D^{s-\frac{1}{2}}\left(|u|^{2 \sigma} \partial_{x} u\right)\right\|_{L_{x}^{1} L_{t}^{2}(\mathbb{R} \times[0, T])}$ is already estimated in (4.11). So we only need to consider the term $\left\|D^{s-\frac{1}{2}}\left(|u|^{2 \sigma} \partial_{x} u\right)\right\|_{L_{t}^{1} L_{x}^{2}([0, T] \times \mathbb{R})}$. Now we claim that

$$
\left\|D^{s-\frac{1}{2}}\left(|u|^{2 \sigma} \partial_{x} u\right)\right\|_{L_{t}^{1} L_{x}^{2}([0, T] \times \mathbb{R})} \lesssim\|u\|_{X_{T}^{2 \sigma+1}}
$$

Again, we split it into two cases: $s=\frac{1}{2}$ and $\frac{1}{2}<s \leq 1$.

Case 1: $s=\frac{1}{2}$.

The term $\left\||u|^{2 \sigma} \partial_{x} u\right\|_{L_{t}^{1} L_{x}^{2}([0, T] \times \mathbb{R})}$ is already estimated in (4.4).

Case 2: $\frac{1}{2}<s \leq 1$.

Using a similar treatment as (4.5), we have

$$
\left\|D^{s-\frac{1}{2}}\left(|u|^{2 \sigma} \partial_{x} u\right)\right\|_{L_{t}^{1} L_{x}^{2}([0, T] \times \mathbb{R})}=\left\|D^{s-\frac{1}{2}}\left(|u|^{2} \cdot|u|^{2 \sigma-2} \partial_{x} u\right)\right\|_{L_{t}^{1} L_{x}^{2}([0, T] \times \mathbb{R})} .
$$

Further, using the Leibniz rule for fractional derivative (2.8), we have

$$
\begin{aligned}
\left\|D^{s-\frac{1}{2}}\left(|u|^{2} \cdot|u|^{2 \sigma-2} \partial_{x} u\right)\right\|_{L_{x}^{2}(\mathbb{R})} \lesssim & \left\|D^{s-\frac{1}{2}}\left(|u|^{2}\right)\right\|_{L_{x}^{\infty}(\mathbb{R})} \cdot\left\||u|^{2 \sigma-2} \partial_{x} u\right\|_{L_{x}^{2}(\mathbb{R})} \\
& +\|u\|_{L_{x}^{\infty}(\mathbb{R})}^{2} \cdot\left\|D^{s-\frac{1}{2}}\left(|u|^{2 \sigma-2} \partial_{x} u\right)\right\|_{L_{x}^{2}(\mathbb{R})},
\end{aligned}
$$

then

$$
\begin{aligned}
& \quad\left\|D^{s-\frac{1}{2}}\left(|u|^{2 \sigma} \partial_{x} u\right)\right\|_{L_{t}^{1} L_{x}^{2}([0, T] \times \mathbb{R})} \cdot\left\||u|^{2 \sigma-2} \partial_{x} u\right\|_{L_{x}^{2} L_{t}^{2}(\mathbb{R} \times[0, T])} \\
& \lesssim\left\|D^{s-\frac{1}{2}}\left(|u|^{2}\right)\right\|_{L_{t}^{2} L_{x}^{\infty}([0, T] \times \mathbb{R})} \cdot\|\|\left\|_{L_{t}^{4} L_{x}^{\infty}([0, T] \times \mathbb{R})} \cdot\right\| D^{s-\frac{1}{2}}\left(|u|^{2 \sigma-2} \partial_{x} u\right) \|_{L_{x}^{2} L_{t}^{2}(\mathbb{R} \times[0, T])} \\
& \quad+\| u{ }^{2}
\end{aligned}
$$


Note that the term $\left\||u|^{2 \sigma-2} \partial_{x} u\right\|_{L_{x}^{2} L_{t}^{2}(\mathbb{R} \times[0, T])}$ has been considered in (4.7), so we only need to deal with the terms $\left\|D^{s-\frac{1}{2}}\left(|u|^{2}\right)\right\|_{L_{t}^{2} L_{x}^{\infty}([0, T] \times \mathbb{R})}$ and $\left\|D^{s-\frac{1}{2}}\left(|u|^{2 \sigma-2} \partial_{x} u\right)\right\|_{L_{x}^{2} L_{t}^{2}(\mathbb{R} \times[0, T])}$ respectively.

For the term $\left\|D^{s-\frac{1}{2}}\left(|u|^{2}\right)\right\|_{L_{t}^{2} L_{x}^{\infty}([0, T] \times \mathbb{R})}$, by (2.8) and the Hölder inequality, we have

$$
\begin{aligned}
\left\|D^{s-\frac{1}{2}}\left(|u|^{2}\right)\right\|_{L_{t}^{2} L_{x}^{\infty}([0, T] \times \mathbb{R})} & \lesssim\|u\|_{L_{t}^{4} L_{x}^{\infty}([0, T] \times \mathbb{R})} \cdot\left\|D^{s-\frac{1}{2}} u\right\|_{L_{t}^{4} L_{x}^{\infty}([0, T] \times \mathbb{R})} \\
& \lesssim\|u\|_{X_{T}}^{2} .
\end{aligned}
$$

For the term $\left\|D^{s-\frac{1}{2}}\left(|u|^{2 \sigma-2} \partial_{x} u\right)\right\|_{L_{x}^{2} L_{t}^{2}(\mathbb{R} \times[0, T])}$, we claim that

$$
\left\|D^{s-\frac{1}{2}}\left(|u|^{2 \sigma-2} \partial_{x} u\right)\right\|_{L_{x}^{2} L_{t}^{2}(\mathbb{R} \times[0, T])} \lesssim\|u\|_{X_{T}}^{2 \sigma-1} .
$$

Indeed, using the Leibniz rule for fractional derivative (2.10), we obtain

$$
\begin{aligned}
& \left\|D^{s-\frac{1}{2}}\left(|u|^{2 \sigma-2} \partial_{x} u\right)\right\|_{L_{x}^{2} L_{t}^{2}(\mathbb{R} \times[0, T])} \\
& \lesssim\left\|D^{s-\frac{1}{2}}\left(|u|^{2 \sigma-2}\right) \cdot \partial_{x} u\right\|_{L_{x}^{2} L_{t}^{2}(\mathbb{R} \times[0, T])}+\left\||u|^{2 \sigma-2} \cdot D^{s-\frac{1}{2}} \partial_{x} u\right\|_{L_{x}^{2} L_{t}^{2}(\mathbb{R} \times[0, T])} \\
& +\left\|D^{s-\frac{1}{2}}\left(|u|^{2 \sigma-2}\right)\right\|_{L_{x}^{2+} L_{t}^{\infty-}(\mathbb{R} \times[0, T])} \cdot\left\|\partial_{x} u\right\|_{L_{x}^{\infty-} L_{t}^{2+}(\mathbb{R} \times[0, T])} .
\end{aligned}
$$

To the term $\left\|D^{s-\frac{1}{2}}\left(|u|^{2 \sigma-2}\right) \cdot \partial_{x} u\right\|_{L_{x}^{2} L_{t}^{2}(\mathbb{R} \times[0, T])}$, using the Hölder inequality, (2.9) and (4.17), note that $4(2 \sigma-3) \geq 4$, we have

$$
\begin{aligned}
&\left\|D^{s-\frac{1}{2}}\left(|u|^{2 \sigma-2}\right) \cdot \partial_{x} u\right\|_{L_{x}^{2} L_{t}^{2}(\mathbb{R} \times[0, T])} \\
& \lesssim\|\| D^{s-\frac{1}{2}}\left(|u|^{2 \sigma-2}\right)\left\|_{L_{t}^{\infty-}([0, T])} \cdot\right\| \partial_{x} u\left\|_{L_{t}^{2+}([0, T])}\right\|_{L_{x}^{2}(\mathbb{R})} \\
& \lesssim\left\|D^{s-\frac{1}{2}}\left(|u|^{2 \sigma-2}\right)\right\|_{L_{x}^{2+} L_{t}^{\infty-}(\mathbb{R} \times[0, T])} \cdot\left\|\partial_{x} u\right\|_{L_{x}^{\infty-} L_{t}^{2+}(\mathbb{R} \times[0, T])} \cdot\left\|D^{s-\frac{1}{2}} u\right\|_{L_{x}^{4+} L_{t}^{\infty-}(\mathbb{R} \times[0, T])} \cdot\|u\|_{X_{T}} \\
& \lesssim\left\||u|^{2 \sigma-3}\right\|_{L_{x}^{4} L_{t}^{\infty}(\mathbb{R} \times[0, T])} \cdot\|\|_{X_{T}} \cdot\left\|D^{s-\frac{1}{2}} u\right\|_{L_{x}^{4+} L_{t}^{\infty-}(\mathbb{R} \times[0, T])} \cdot \\
& \lesssim\|u\|^{2 \sigma-2}
\end{aligned}
$$

Hence, by (4.15), we get

$$
\left\|D^{s-\frac{1}{2}}\left(|u|^{2 \sigma-2}\right) \cdot \partial_{x} u\right\|_{L_{x}^{2} L_{t}^{2}(\mathbb{R} \times[0, T])} \lesssim\|u\|_{X_{T}}^{2 \sigma-1} .
$$

To the term $\left\||u|^{2 \sigma-2} \cdot D^{s-\frac{1}{2}} \partial_{x} u\right\|_{L_{x}^{2} L_{t}^{2}(\mathbb{R} \times[0, T])}$, using Hölder's inequality, note that $2(2 \sigma-2) \geq 4$, we get

$$
\begin{aligned}
\left\||u|^{2 \sigma-2} \cdot D^{s-\frac{1}{2}} \partial_{x} u\right\|_{L_{x}^{2} L_{t}^{2}(\mathbb{R} \times[0, T])} & \lesssim\|u\|_{L_{x}^{2(2 \sigma-2)} L_{t}^{\infty}(\mathbb{R} \times[0, T])}^{2 \sigma-2} \cdot\left\|D^{s-\frac{1}{2}} \partial_{x} u\right\|_{L_{x}^{\infty} L_{t}^{2}(\mathbb{R} \times[0, T])} \\
& \lesssim\|u\|_{X_{T}}^{2 \sigma-1} .
\end{aligned}
$$


To the term $\left\|D^{s-\frac{1}{2}}\left(|u|^{2 \sigma-2}\right)\right\|_{L_{x}^{2+} L_{t}^{\infty-}(\mathbb{R} \times[0, T])} \cdot\left\|\partial_{x} u\right\|_{L_{x}^{\infty-} L_{t}^{2+}(\mathbb{R} \times[0, T])}$, as the same estimation in (4.26), we have

$$
\left\|D^{s-\frac{1}{2}}\left(|u|^{2 \sigma-2}\right)\right\|_{L_{x}^{2+} L_{t}^{\infty-}(\mathbb{R} \times[0, T])} \cdot\left\|\partial_{x} u\right\|_{L_{x}^{\infty-} L_{t}^{2+}(\mathbb{R} \times[0, T])} \lesssim\|u\|_{X_{T}}^{2 \sigma-1} .
$$

Thus we finish the proof of claim (4.24) and (4.25) and obtain

$$
\left\|D^{s-\frac{1}{2}} u\right\|_{L_{x}^{4} L_{t}^{\infty}(\mathbb{R} \times[0, T])} \lesssim\|\varphi\|_{H_{x}^{s}(\mathbb{R})}+\|u\|_{X_{T}}^{2 \sigma+1} .
$$

4.6. Estimates on $\left\|D^{s-\frac{1}{2}} \partial_{x} u\right\|_{L_{x}^{\infty} L_{t}^{2}(\mathbb{R} \times[0, T])}$. Using Duhamel's formula (4.2) and the smoothing effects (2.3) and (2.5), we get

$$
\begin{aligned}
\left\|D^{s-\frac{1}{2}} \partial_{x} u\right\|_{L_{x}^{\infty} L_{t}^{2}(\mathbb{R} \times[0, T])} & \left\|e^{i t \Delta} D^{s-\frac{1}{2}} \partial_{x} \varphi\right\|_{L_{x}^{\infty} L_{t}^{2}(\mathbb{R} \times[0, T])} \\
& +\left\|\partial_{x} \int_{0}^{t} e^{i\left(t-t^{\prime}\right) \Delta} D^{s-\frac{1}{2}}\left(|u|^{2 \sigma} \partial_{x} u\right)\left(t^{\prime}\right) \mathrm{d} t^{\prime}\right\|_{L_{x}^{\infty} L_{t}^{2}(\mathbb{R} \times[0, T])} \\
\lesssim & \left\|D^{s} \varphi\right\|_{L_{x}^{2}(\mathbb{R})}+\left\|D^{s-\frac{1}{2}}\left(|u|^{2 \sigma} \partial_{x} u\right)\right\|_{L_{x}^{1} L_{t}^{2}(\mathbb{R} \times[0, T])} \\
& \lesssim\|\varphi\|_{H_{x}^{s}(\mathbb{R})}+\left\|D^{s-\frac{1}{2}}\left(|u|^{2 \sigma} \partial_{x} u\right)\right\|_{L_{x}^{1} L_{t}^{2}(\mathbb{R} \times[0, T])}
\end{aligned}
$$

Note that we already have the estimation on $\left\|D^{s-\frac{1}{2}}\left(|u|^{2 \sigma} \partial_{x} u\right)\right\|_{L_{x}^{1} L_{t}^{2}(\mathbb{R} \times[0, T])}$ in (4.21). Then

$$
\left\|D^{s-\frac{1}{2}} \partial_{x} u\right\|_{L_{x}^{\infty} L_{t}^{2}(\mathbb{R} \times[0, T])} \lesssim\|\varphi\|_{H_{x}^{s}(\mathbb{R})}+\|u\|_{X_{T}}^{2 \sigma+1}
$$

4.7. Estimates on $\left\|D^{s-\frac{1}{2}} u\right\|_{L_{t}^{4} L_{x}^{\infty}([0, T] \times \mathbb{R})^{\cdot}} \quad$ By Duhamel's formula (4.2) and the Strichartz estimates (2.1) and (2.2), we have

$$
\begin{aligned}
\left\|D^{s-\frac{1}{2}} u\right\|_{L_{t}^{4} L_{x}^{\infty}([0, T] \times \mathbb{R})} \lesssim & \left\|e^{i t \Delta} D^{s-\frac{1}{2}} \varphi\right\|_{L_{t}^{4} L_{x}^{\infty}([0, T] \times \mathbb{R})} \\
& +\left\|\int_{0}^{t} e^{i\left(t-t^{\prime}\right) \Delta} D^{s-\frac{1}{2}}\left(|u|^{2 \sigma} \partial_{x} u\right)\left(t^{\prime}\right) \mathrm{d} t^{\prime}\right\|_{L_{t}^{4} L_{x}^{\infty}([0, T] \times \mathbb{R})} \\
& \lesssim\left\|D^{s-\frac{1}{2}} \varphi\right\|_{L_{x}^{2}(\mathbb{R})}+\left\|D^{s-\frac{1}{2}}\left(|u|^{2 \sigma} \partial_{x} u\right)\right\|_{L_{t}^{1} L_{x}^{2}([0, T] \times \mathbb{R})} \\
& \lesssim\|\varphi\|_{H_{x}^{s}(\mathbb{R})}+\left\|D^{s-\frac{1}{2}}\left(|u|^{2 \sigma} \partial_{x} u\right)\right\|_{L_{t}^{1} L_{x}^{2}([0, T] \times \mathbb{R})} .
\end{aligned}
$$

Note that the estimation on $\left\|D^{s-\frac{1}{2}}\left(|u|^{2 \sigma} \partial_{x} u\right)\right\|_{L_{t}^{1} L_{x}^{2}([0, T] \times \mathbb{R})}$ is obtained in (4.24). Then we have

$$
\left\|D^{s-\frac{1}{2}} u\right\|_{L_{t}^{4} L_{x}^{\infty}([0, T] \times \mathbb{R})} \lesssim\|\varphi\|_{H_{x}^{s}(\mathbb{R})}+\|u\|_{X_{T}}^{2 \sigma+1}
$$

Finally, all the estimates on $\|u\|_{X_{T}}$ are obtained and we have

$$
\|u\|_{X_{T}} \lesssim\|\varphi\|_{H_{x}^{s}(\mathbb{R})}+\|u\|_{X_{T}}^{2 \sigma+1}
$$

uniformly on $T$. Hence we get $\|u\|_{X_{\infty}} \lesssim\|\varphi\|_{H_{x}^{s}(\mathbb{R})}$, which gives the proof of the global well-posedness. 
Next we prove the scattering statement. Set

$$
u_{+}=\varphi-\int_{0}^{+\infty} e^{-i t^{\prime} \Delta}\left(|u|^{2 \sigma} \partial_{x} u\right) \mathrm{d} t^{\prime} .
$$

Using Duhamel's formula (4.2), we have

$$
u(t)-e^{i t \Delta} u_{+}=\int_{t}^{+\infty} e^{i\left(t-t^{\prime}\right) \Delta}\left(|u|^{2 \sigma} \partial_{x} u\right) \mathrm{d} t^{\prime}
$$

By interpolation, for any $0 \leq s^{\prime}<s$, we have that for some $\theta \in[0,1)$,

$$
\left\|u(t)-e^{i t \Delta} u_{+}\right\|_{H^{s^{\prime}}(\mathbb{R})} \lesssim\left\|u(t)-e^{i t \Delta} u_{+}\right\|_{L^{2}(\mathbb{R})}^{\theta} \cdot\left\|u(t)-e^{i t \Delta} u_{+}\right\|_{\dot{H}_{x}^{s}(\mathbb{R})}^{1-\theta} .
$$

For the term $\left\|u(t)-e^{i t \Delta} u_{+}\right\|_{L^{2}(\mathbb{R})}$, by (4.8), we have

$$
\begin{aligned}
\left\|u(t)-e^{i t \Delta} u_{+}\right\|_{L^{2}(\mathbb{R})} & \lesssim\left\||u|^{2 \sigma} \partial_{x} u\right\|_{L_{t}^{1} L_{x}^{2}([t,+\infty] \times \mathbb{R})} \\
& \lesssim\|u\|_{L_{t}^{4} L_{x}^{\infty}([t,+\infty] \times \mathbb{R})}^{2} \cdot\|u\|_{X_{\infty}}^{2 \sigma-1}
\end{aligned}
$$

Since $\|u\|_{X_{\infty}} \lesssim\|\varphi\|_{H_{x}^{s}(\mathbb{R})}$, we get

$$
\|u\|_{L_{t}^{4} L_{x}^{\infty}([t,+\infty] \times \mathbb{R})} \rightarrow 0, \quad \text { when } \quad t \rightarrow+\infty .
$$

Therefore

$$
\left\|u(t)-e^{i t \Delta} u_{+}\right\|_{L^{2}(\mathbb{R})} \rightarrow 0, \quad \text { when } t \rightarrow+\infty .
$$

For the term $\left\|u(t)-e^{i t \Delta} u_{+}\right\|_{\dot{H}_{x}^{s}(\mathbb{R})}$, by smoothing effects (2.4) and (4.11), we have

$$
\begin{aligned}
\left\|u(t)-e^{i t \Delta} u_{+}\right\|_{\dot{H}_{x}^{s}(\mathbb{R})} & \lesssim\left\|D^{\frac{1}{2}} \int_{t}^{+\infty} e^{i\left(t-t^{\prime}\right) \Delta} D^{s-\frac{1}{2}}\left(|u|^{2 \sigma} \partial_{x} u\right) \mathrm{d} t^{\prime}\right\|_{L^{2}(\mathbb{R})} \\
& \lesssim\left\|D^{s-\frac{1}{2}}\left(|u|^{2 \sigma} \partial_{x} u\right)\right\|_{L_{x}^{1} L_{t}^{2}(\mathbb{R} \times[0,+\infty])} \\
& \lesssim\|u\|_{X_{\infty}}^{2 \sigma+1} .
\end{aligned}
$$

Hence, we have

$$
\left\|u(t)-e^{i t \Delta} u_{+}\right\|_{\dot{H}_{x}^{s}(\mathbb{R})} \lesssim\|\varphi\|_{H_{x}^{s}(\mathbb{R})}^{2 \sigma+1} .
$$

Combining the estimates (4.27), (4.28) and (4.29), we get

$$
\left\|u(t)-e^{i t \Delta} u_{+}\right\|_{H^{s^{\prime}}(\mathbb{R})} \rightarrow 0, \quad \text { as } t \rightarrow+\infty .
$$

This proves the scattering statement and thus finish the proof of the Theorem 1.2 .

\section{ACKNOWLEDGEMENTS}

The authors are grateful to the referee who gave useful notes on grammatical/typographical errors and many helpful comments and suggestions. 


\section{REFERENCES}

[1] Bejenaru, I., Quadratic nonlinear derivative Schrödinger equations, I. Int. Math. Res. Pap., Art. ID 70630 (2006), 84 pp.

[2] Bejenaru, I., Quadratic nonlinear derivative Schrödinger equations, II. Trans. Amer. Math. Soc., 360 (11) (2008), 5925-5957.

[3] Biagioni, H., Linares, F., Ill-posedness for the derivative Schrödinger and generalized Benjamin-Ono equations, Trans. Amer. Math. Soc., 353 (9) (2001), 3649-3659 .

[4] Bourgain, J., Li, D., On an endpoint Kato-Ponce inequality, Differential Integral Equations, 27 (11-12) (2014), 1037-1072.

[5] Cazenave, T., Semilinear Schrödinger equations, American Mathematical Society, 2003.

[6] Cazenave, T., Weissler, F, Rapidly decaying solutions of the nonlinear Schrödinger equation. Comm. Math. Phys., 147 (1992), 75-100.

[7] Cher, Y., Simpson, G., Sulem, C., Local structure of singular profiles for a derivative nonlinear Schrödinger equation, SIAM J. Appl. Dyn. Syst., 16 (1) (2017), 514-545.

[8] Colin, M., Ohta, M., Stability of solitary waves for derivative nonlinear Schrödinger equation, Ann. Inst. H. Poincaré Anal. Non Linéaire, 23 (5) (2006), 753-764.

[9] Colliander, J., Keel, M., Staffilani, G., Takaoka, H., Tao, T., Global well-posedness for Schrödinger equations with derivative, SIAM J. Math. Anal., 33 (3) (2001), 649-669.

[10] Colliander, J., Keel, M., Staffilani, G., Takaoka, H., Tao, T., A refined global well-posedness for Schrödinger equations with derivative, SIAM J. Math. Anal., 34 (1) (2002), 64-86.

[11] Chihara, H., Gain of regularity for semilinear Schrödinger equations, Math. Ann., 315 (4) (1999), 529567.

[12] Cohn, S., Resonance and long time existence for the quadratic semilinear Schrödinger equation, Comm. Pure Appl. Math., 45 (8) (1992) 973-1001.

[13] Dan, Y., Li, Y., Ning, L., Regularity and uniqueness for the rough solutions of the derivative nonlinear Schrödinger equation, Differ. Integral Equ., 32 (56) (2019) 265-290.

[14] Delort, J.-M., Global Solutions for Small Nonlinear Long Range Perturbations of Two Dimensional Schrödinger Equations, Mém. Soc. Math. Fr. (N.S.), vol. 91, 2002.

[15] Fukaya, N., Hayashi, M., Inui, T., A sufficient condition for global existence of solutions to a generalized derivative nonlinear Schrödinger equation, Anal. PDE, 10 (5) (2017), 1149-1167.

[16] Fukaya, N., Instability of solitary waves for a generalized derivative nonlinear Schrödinger equation in a borderline case, Kodai Math. J., 40 (3) (2017), 450-467.

[17] Ginibre, J., Ozawa, T., Velo, G., On the existence of the wave operators for a class of nonlinear Schrödinger equations. Ann. Inst. H. Poincaré Phys. Théor. 60 (1994), no. 2, 211-239.

[18] Guo, B., Wu, Y., Orbital stability of solitary waves for the nonlinear derivative Schrödinger equation, J. Differential Equations, 123 (1) (1995), 35-55.

[19] Guo, B., Tan, S., On smooth solutions to the initial value problem for the mixed nonlinear Schrödinger equations, Proc. Roy. Soc. Edinburgh Sect, A, 119 (1-2) (1991), 31-45.

[20] Guo, Q., Orbital stability of solitary waves for generalized derivative nonlinear Schrödinger equations in the endpoint case, Ann. Henri Poincaré, 19 (9) (2018), 2701-2715.

[21] Guo, Z., Wu, Y., Global well-posedness for the derivative nonlinear Schrödinger equation in $H^{\frac{1}{2}}(\mathbb{R})$, Discrete Contin. Dyn. Syst., 37 (1) (2017), 257-264.

[22] Guo, Z., Ning, C. and Wu, Y., Instability of the solitary wave solutions for the generalized derivative nonlinear Schrödinger equation in the critical frequency case, arXiv:1803.07700

[23] Guo, Z., Hayashi, N., Lin, Y., Naumkin, P., Modified scattering operator for the derivative nonlinear Schrödinger equation. SIAM J. Math. Anal., 45 (6) (2013), 3854-3871.

[24] Germain, P., Masmoudi, N., and Shatah, J., Global solutions for 2D quadratic Schrödinger equation, J. Math. Pures Appl., 97 (2012) 505-543

[25] Grünrock, A., Bi- and trilinear Schrödinger estimates in one space dimension with applications to cubic NLS and DNLS. Int. Math. Res. Not., 41 (2005), 2525-2558 .

[26] Grünrock, A., Herr, S., Low regularity local well-posedness of the derivative nonlinear Schrödinger equation with periodic initial data, SIAM J. Math. Anal., 39 (6) (2008), 1890-1920 .

[27] Grünrock, A., On the Cauchy- and periodic boundary value problem for a certain class of derivative nonlinear Schrödinger equations, arXiv:math/0006195 
[28] Hao, C., Well-posedness for one dimensional derivative nonlinear Schrödinger equations, Commun. Pure Appl. Anal., 6 (4) (2007), 997-1021.

[29] N. Hayashi, C. Miao, P. Naumkin, Global existence of small solutions to the generalized derivative nonlinear Schrödinger equation, Asymptot. Anal. 21 (2) (1999) 133-147.

[30] Hayashi, N., Ozawa, T., On the derivative nonlinear Schrödinger equation, Phys. D., 55 (1-2) (1992), $14-36$.

[31] Hayashi, N., Ozawa, T., Finite energy solutions of nonlinear Schrödinger equations of derivative type, SIAM J. Math. Anal., 25 (6) (1994), 1488-1503.

[32] Hayashi, N., Ozawa, T., Modified wave operators for the derivative nonlinear Schrödinger equation, Math. Ann., 298 (3) (1994), 557-576.

[33] Hayashi, N., Ozawa, T., Well-posedness for a generalized derivative nonlinear Schrödinger equation, J. Differential Equations, 261 (10) (2016), 5424-5445.

[34] Herr, S., On the Cauchy problem for the derivative nonlinear Schrödinger equation with periodic boundary condition, Int. Math. Res. Not. Art. ID 96763 (2006), 33 pp.

[35] Hirayama, H., Well-posedness and scattering for nonlinear Schrödinger equations with a derivative nonlinearity at the scaling critical regularity, Funkcial. Ekvac., 58 (3) (2015), 431-450.

[36] Jenkins, R., Liu, J., Perry, P., and Sulem. C., Global well-posedness for the derivative non-linear Schrdinger equation, Comm. Partial Differential Equations, 43 (8) (2018), 1151-1195.

[37] Jenkins, R., Liu, J., Perry, P. and Sulem. C., The derivative nonlinear Schrdinger equation: global well-posedness and solton resolution, Quart. Appl. Math., 78 (1) (2020), 33-73.

[38] Kwon, S., Wu, Y., Orbital stability of solitary waves for derivative nonlinear Schrödinger equation, J. Anal. Math. 135 (2) (2018), 473-486.

[39] Kenig, C., Ponce, G., and Vega, L., Oscillatory integrals and regularity of dispersive equations, Indiana Univ. Math. J., 40 (1) (1991), 33-69.

[40] Kenig, C., Ponce, G., and Vega, L., Well-posedness and scattering results for the generalized Kortewegde Vries equation via contraction principle, Comm. Pure Appl. Math., 46 (4) (1993), 527-620.

[41] Kenig, C., Ponce, G., and Vega, L., Small solutions to nonlinear Schrödinger equations, Ann. Inst. H. Poincaré Anal. Non Linéaire, 10 (3) (1993), 255-288.

[42] Kenig, C., Ruiz, A., A strong type $(2,2)$ estimate for the maximal operator associated to the Schrödinger equation, Trans. Amer. Math. Soc., 280 (1) (1983), 239-246.

[43] Le Coz, S., Wu, Y., Stability of multi-solitons for the derivative nonlinear schrödinger equation, Int. Math. Res. Not., 13 (2018), 4120-4170.

[44] Li, B., Ning, C., Instability of the solitary wave solutions for the generalized derivative nonlinear schrödinger equation in the endpoint case, arXiv:1804.02738.

[45] Li, D., On Kato-Ponce and fractional Leibniz, Rev. Mat. Iberoam, 35 (1) (2019), 23-100.

[46] Linares, F., Ponce, G., and Santos, G. N., On a class of solutions to the generalized derivative Schrödinger equations, Acta Math. Sin. (Engl. Ser.), 35 (6) (2019), 1057-1073.

[47] Linares, F., Ponce, G., and Santos, G. N., On a class of solutions to the generalized derivative Schrödinger equations II, J. Differential Equations, 267 (1) (2019), 97-118.

[48] Linares, F., Ponce, G., Introduction to Nonlinear Dispersive Equations, Springer, 2009.

[49] Liu, X., Simpson, G., Sulem, C., Stability of solitary waves for a generalized derivative nonlinear Schrödinger equation, J. Nonlinear Sci., 23 (4) (2013), 557-583.

[50] Liu, J., Perry, P., Sulem, C. Global existence for the derivative nonlinear Schrödinger equation by the method of inverse scattering, Comm. Partial Differential Equations, 41 (11) (2016), 1692-1760.

[51] Liu, J., Perry, P., Sulem, C., Long-time behavior of solutions to the derivative nonlinear Schrödinger equation for soliton-free initial data, Ann. Inst. H. Poincaré Anal. Non Linéaire, 35 (1) (2018), $217-265$.

[52] Liu, X., Simpson, G., Sulem, C., Focusing singularity in a derivative nonlinear Schrödinger equation, Phys. D., 262 (2013), 48-58.

[53] Miao, C., Tang, X., Xu, G., Stability of the traveling waves for the derivative Schrödinger equation in the energy space. Calc. Var. Partial Differential Equations, 56 (2) (2017), Art. 45, 48 pp.

[54] Miao, C., Tang, X., Xu, G., Solitary waves for nonlinear Schrödinger equation with derivative, Commun. Contemp. Math., 20 (4) (2018), 1750049, 27 pp.

[55] Mio, W., Ogino, T., Minami, K., and Le Takeda, S., Modified nonlinear Schrödinger for Alfvén waves propagating along the magnetic field in cold plasmas, J. Phys. Soc. Japan, 41 (1) (1976), $265-271$. 
[56] Molinet, L., Ribaud, F., Well-posedness results for the generalized Benjamin-Ono equation with small initial data, J. Math. Pures Appl. (9), 83 (2) (2004), 277-311.

[57] Mosincat, R., Global well-posedness of the derivative nonlinear Schrödinger equation with periodic boundary condition in $H^{\frac{1}{2}}$. J. Differential Equations, 263 (8) (2017), 4658-4722.

[58] Mosincat, R., Oh, T., A remark on global well-posedness of the derivative nonlinear Schrödinger equation on the circle. C. R. Math. Acad. Sci. Paris, 353 (9) (2015), 837-841.

[59] Mosincat R., Yoon H., Unconditional uniqueness for the derivative nonlinear Schrödinger equation on the real line, Discrete Contin. Dyn. Syst., 40 (1) (2020), 47-80.

[60] Miao, C., Wu, Y., Xu, G., Global well-posedness for Schrödinger equation with derivative in $H^{\frac{1}{2}}(\mathbb{R})$, J. Differential Equations, 251 (8) (2011), 2164-2195.

[61] Nakanishi, K, Asymptotically-free solutions for the short-range nonlinear Schrödinger equation, SIAM J. Math. Anal., 32 (2001), 1265-1271.

[62] Pelinovsky D., and Shimabukuro, Y., Existence of global solutions to the derivative NLS equation with the inverse scattering transform method, Int. Math. Res. Not, 18 (2018), 5663-5728.

[63] Pelinovsky, D., Saalmann, A., and Shimabukuro, Y., The derivative NLS equation: global existence with solitons. Dyn. Partial Differ. Equ., 14 (3) (2017), 217-294.

[64] Pornnopparath, D., Small data well-posedness for derivative nonlinear Schrödinger equations, J. Differential Equations, 265 (8) (2018), 3792-3840.

[65] Rogers, K., Villarroya, P., Global estimates for the Schrödinger maximal operator, Ann. Acad. Sci. Fenn. Math., 32 (2) (2007), 425-435.

[66] Santos, G. N., Existence and uniqueness of solution for a generalized nonlinear derivative Schrödinger equation, J. Differential Equations, 259 (5) (2015), 2030-2060.

[67] Strauss, W. A., Nonlinear scattering theory at low energy. J. Funct. Anal. 41 (1981), no. 1, $110-133$.

[68] Tang, X., and Xu, G., Stability of the sum of two solitary waves for (gDNLS) in the energy space, J. Differential Equations, 264 (6) (2018), 4094-4135.

[69] Takaoka, H., Well-posedness for the one dimensional nonlinear Schrödinger equation with the derivative nonlinearity, Adv. Differential Equations, 4 (4) (1999), 561-580.

[70] Takaoka, H., Global well-posedness for Schrödinger equations with derivative in a nonlinear term and data in low-order Sobolev spaces, Electron. J. Differential Equations, 42 (2001), 23 pp.

[71] Takaoka, H., A priori estimates and weak solutions for the derivative nonlinear Schrödinger equation on torus below $H^{1 / 2}$, J. Differential Equations, 260 (1) (2016), 818-859.

[72] Tsutsumi, M., Fukuda, I., On solutions of the derivative nonlinear Schrödinger equation. Existence and Uniqueness Theorem. Funkcial. Ekvac., 23 (1980), 259-277.

[73] Wang, Y., Global well-posedness and scattering for derivative Schrödinger equation, Comm. Partial Differential Equations, 36 (10) (2011), 1694-1722.

[74] Wu, Y. F., Global well-posedness for the nonlinear Schrödinger equation with derivative in energy space, Anal. PDE, 6 (8) (2013), 1989-2002.

[75] Wu, Y. F., Global well-posedness on the derivative nonlinear Schrödinger equation, Anal. PDE, 8 (5) (2015), 1101-1112.

Center for Applied Mathematics, Tianjin University, Tianjin 300072, China

E-mail address: baimaths@hotmail.com

Center for Applied Mathematics, Tianjin University, Tianjin 300072, China

E-mail address: yerfmath@gmail.com

Department of Mathematical Sciences, Norwegian University of Science and Technology, TrondheIM 7491, NorWAY

E-mail address: jxuemath@hotmail.com 